\title{
Violations of the equivalence principle in a dilaton-runaway scenario
}

\author{
T. Damour, ${ }^{1}$ F. Piazza, ${ }^{2}$ and G. Veneziano ${ }^{3,4}$ \\ ${ }^{1}$ Institut des Hautes Etudes Scientifiques, 91440 Bures-sur-Yvette, France \\ ${ }^{2}$ Dipartimento di Fisica, Università di Milano Bicocca, Piazza delle Scienze 3, I-20126 Milan, Italy \\ ${ }^{3}$ Theory Division, CERN, CH-1211 Geneva 23, Switzerland \\ ${ }^{4}$ Laboratoire de Physique Théorique, Université Paris Sud, 91405 Orsay, France
}

(Received 13 May 2002; published 28 August 2002)

\begin{abstract}
We explore a version of the cosmological dilaton-fixing and decoupling mechanism in which the dilaton dependence of the low-energy effective action is extremized for infinitely large values of the bare string coupling $g_{s}^{2}=e^{\phi}$. We study the efficiency with which the dilaton $\phi$ runs away toward its "fixed point" at infinity during a primordial inflationary stage, and thereby approximately decouples from matter. The residual dilaton couplings are found to be related to the amplitude of the density fluctuations generated during inflation. For the simplest inflationary potential $V(\chi)=\frac{1}{2} m_{\chi}^{2}(\phi) \chi^{2}$, the residual dilaton couplings are shown to predict violations of the universality of gravitational acceleration near the $\Delta a / a \sim 10^{-12}$ level. This suggests that a modest improvement in the precision of equivalence principle tests might be able to detect the effect of such a runaway dilaton. Under some assumptions about the coupling of the dilaton to dark matter and/or dark energy, the expected time variation of natural "constants" (in particular of the fine-structure constant) might also be large enough to be within reach of improved experimental or observational data.
\end{abstract}

DOI: 10.1103/PhysRevD.66.046007

PACS number(s): 11.25. - w, 04.80.Cc, 98.80.Cq

\section{INTRODUCTION}

All string theory models predict the existence of a scalar partner of the spin 2 graviton: the dilaton $\phi$, whose vacuum expectation value (VEV) determines the string coupling constant $g_{s}=e^{\phi / 2}[1]$. At tree level, the dilaton is massless and has gravitational-strength couplings to matter which violate the equivalence principle [2]. This is in violent conflict with present experimental tests of general relativity. It is generally assumed that this conflict is avoided because, after supersymmetry breaking, the dilaton might acquire a (large enough) mass (say $m_{\phi} \gtrsim 10^{-3} \mathrm{eV}$ so that observable deviations from Einstein's gravity are quenched at distances larger than a fraction of a millimeter). However, Ref. [3] (see also [4]) has proposed a mechanism which can naturally reconcile a massless dilaton with existing experimental data. The basic idea of Ref. [3] was to exploit the string-loop modifications of the (four-dimensional) effective low-energy action (we use the signature -+++ )

$$
\begin{aligned}
S= & \int d^{4} x \sqrt{\tilde{g}}\left(\frac{B_{g}(\phi)}{\alpha^{\prime}} \widetilde{R}+\frac{B_{\phi}(\phi)}{\alpha^{\prime}}\left[2 \tilde{\square} \phi-(\tilde{\nabla} \phi)^{2}\right]\right. \\
& \left.-\frac{1}{4} B_{F}(\phi) \widetilde{F}^{2}-V+\ldots\right),
\end{aligned}
$$

i.e., the $\phi$ dependence of the various coefficients $B_{i}(\phi), i$ $=g, \phi, F, \ldots$, given in the weak-coupling region $\left(e^{\phi} \rightarrow 0\right)$ by series of the form

$$
B_{i}(\phi)=e^{-\phi}+c_{0}^{(i)}+c_{1}^{(i)} e^{\phi}+c_{2}^{(i)} e^{2 \phi}+\cdots,
$$

coming from genus expansion of string theory: $B_{i}$ $=\Sigma_{n} g_{s}^{2(n-1)} c_{n}^{(i)}$, with $n=0,1,2, \ldots$. It was shown in [3] that, if there exists a special value $\phi_{m}$ of $\phi$ which extremizes all the (relevant) coupling functions $B_{i}^{-1}(\phi)$, the cosmological evolution of the graviton-dilaton-matter system naturally drives $\phi$ toward $\phi_{m}$. This provides a mechanism for fixing a massless dilaton at a value where it decouples from matter ("least coupling principle"). A simple situation where the existence of a universally extremizing dilaton value $\phi_{m}$ is guaranteed is that of $S$ duality, i.e., a symmetry $g_{s} \leftrightarrow 1 / g_{s}$, or $\phi \rightarrow-\phi$ (so that $\left.\phi_{m}=0\right)$.

It has been recently suggested [5] that the infinite-barecoupling limit $g_{s} \rightarrow \infty(\phi \rightarrow+\infty)$ might yield smooth finite limits for all the coupling functions, namely,

$$
B_{i}(\phi)=C_{i}+\mathcal{O}\left(e^{-\phi}\right) .
$$

Under this assumption, the coupling functions are all extremized at infinity, i.e., $\phi_{m}=+\infty$. The late-time cosmology of models satisfying Eq. (1.3) has recently been explored [6]. In the "large $N$ "-type toy model of [5] it would be natural to expect that the $\mathcal{O}\left(e^{-\phi}\right)$ term in Eq. (1.3) be positive, so that $B_{i}(\phi)$ be minimized at infinity. This would correspond to couplings $\lambda_{i}(\phi) \sim B_{i}^{-1}(\phi)=C_{i}^{-1}-\mathcal{O}\left(e^{-\phi}\right)$ which are maximized at infinity. Note, however, that the most relevant cosmological coupling for this work, the coupling to the inflaton, $\lambda(\phi)$, contained in $V$ [see Eq. (2.11) below] is closer to a $B_{i}$ than to its inverse. Thus $\lambda(\phi)$ is naturally minimized at infinity (see further discussion of this point below), a crucial property for the attractor mechanism of $[3,4]$.

In this paper $^{1}$ we shall consider in detail the early-time cosmology of models satisfying Eq. (1.3). More precisely, our main aims will be (i) to study the efficiency with which a primordial inflationary stage drives $\phi$ toward the "fixed point" at infinity $\phi_{m}=+\infty$ (thereby generalizing the work

\footnotetext{
${ }^{1}$ The main results of this work were recently summarized in a short work [7].
} 
[8] which considered the inflationary attraction toward a local extremum $\phi_{m}$ ), and (ii) to give quantitative estimates of the present violations of the equivalence principle (nonuniversality of free fall, and variation of "constants"). Our most important conclusion is that the runaway of the dilaton toward strong coupling [under the assumption (1.3)] naturally leads to equivalence-principle violations which are rather large, in the sense of not being much smaller than the presently tested level $\sim 10^{-12}$. This gives additional motivation for the currently planned improved tests of the universality of free fall. Within our scenario, most of the other deviations from general relativity ("post-Einsteinian" effects in gravitationally interacting systems: solar system, binary pulsars, etc.) are too small to be of phenomenological interest. However, under some assumptions about the coupling of $\phi$ to dark matter and/or dark energy, the time variation of the natural "constants" (notably the fine-structure constant) predicted by our scenario might be large enough to be within reach of improved experimental and/or observational data. The phenomenologically interesting conclusion that equivalence-principle violations are generically predicted to be rather large after inflation (in sharp contrast with the results of [8]) is due to the fact that the attraction toward an extremum at infinity is much less effective than the attraction toward a (finite) local extremum as originally contemplated in [3]. This reduced effectiveness was already pointed out in Ref. [4] within the context of equivalence-principlerespecting tensor-scalar theories (in the manner of the Jordan-Fierz-Brans-Dicke theories).

\section{DILATON RUNAWAY}

In this section we study the dilaton's runaway during the various stages of cosmological evolution. We first show (Sec. II A) that, as in the case of a local extremum [8], inflation is particularly efficient in pushing $\phi$ toward the fixed point. We then argue (Sec. II B) that the order of magnitude of the bare string coupling $e^{\phi} \simeq e^{c \varphi}$ does not suffer further appreciable changes during all the subsequent evolution.

\section{A. The inflationary period}

Assuming some primordial inflationary stage driven by the potential energy of an inflaton field $\tilde{\chi}$, and taking into account generic couplings to the dilaton $\phi$, we consider an effective action of the form

$$
\begin{aligned}
S= & \int d^{4} x \sqrt{\tilde{g}}\left(\frac{B_{g}(\phi)}{\alpha^{\prime}} \widetilde{R}+\frac{B_{\phi}(\phi)}{\alpha^{\prime}}\left[2 \tilde{\square} \phi-(\tilde{\nabla} \phi)^{2}\right]\right. \\
& \left.-\frac{1}{2} B_{\chi}(\phi)(\tilde{\nabla} \tilde{\chi})^{2}-\tilde{V}(\tilde{\chi}, \phi)\right) .
\end{aligned}
$$

In this string-frame action, the dilaton dependence of all the functions $B_{i}(\phi), \widetilde{V}(\tilde{\chi}, \phi)$ is assumed to be of the form (1.2). It is convenient to replace the ( $\sigma$-model) string metric $\tilde{g}_{\mu \nu}$ by the conformally related Einstein metric $g_{\mu \nu}$ $=C B_{g}(\phi) \tilde{g}_{\mu \nu}$, and the dilaton field by the variable

$$
\begin{aligned}
\varphi=\int d \phi\left[\frac{3}{4}\left(\frac{B_{g}^{\prime}}{B_{g}}\right)^{2}+\frac{B_{\phi}^{\prime}}{B_{g}}+\frac{1}{2} \frac{B_{\phi}}{B_{g}}\right]^{1 / 2}, \\
B^{\prime} \equiv \partial B / \partial \phi .
\end{aligned}
$$

The normalization constant $C$ is chosen so that the string units coincide with the Einstein units when $\phi \rightarrow+\infty$ : $C B_{g}(+\infty)=1$. [Note that $C=1 / C_{g}$ in terms of the general notation of Eq. (1.3).] Introducing the (modified) Planck mass

$$
\tilde{m}_{P}^{2}=\frac{1}{4 \pi G}=\frac{4}{C \alpha^{\prime}}
$$

and replacing also the inflaton by the dimensionless variable $\chi=C^{-1 / 2} \tilde{m}_{P}^{-1} \tilde{\chi}$, we end up with an action of the form

$$
\begin{aligned}
S= & \int d^{4} x \sqrt{g}\left[\frac{\tilde{m}_{P}^{2}}{4} R-\frac{\tilde{m}_{P}^{2}}{2}(\nabla \varphi)^{2}\right. \\
& \left.-\frac{\tilde{m}_{P}^{2}}{2} F(\varphi)(\nabla \chi)^{2}-\tilde{m}_{P}^{4} V(\chi, \varphi)\right],
\end{aligned}
$$

where

$$
\begin{gathered}
F(\varphi)=B_{\chi}(\phi) / B_{g}(\phi), \\
V(\chi, \varphi)=C^{-2} \tilde{m}_{P}^{-4} B_{g}^{-2}(\phi) \widetilde{V}(\tilde{\chi}, \phi) .
\end{gathered}
$$

In view of our basic assumption (1.3), note that, in the strong-coupling limit $\phi \rightarrow+\infty, d \varphi / d \phi$ tends, according to Eq. (2.2), to the constant $\left(C_{\phi} / 2 C_{g}\right)^{1 / 2}$, while the dilatondependent factor $F(\varphi)$ in front of the inflaton kinetic term tends to the constant $C_{\chi} / C_{g}$. The toy model of Ref. [5] suggests that the various (positive) constants $C_{i}$ in Eq. (1.3) are all largish and comparable to each other. We shall therefore assume that the various ratios $C_{i} / C_{j}$ are of order unity. The most important such ratio for the following is $c$ $\equiv\left(2 C_{g} / C_{\phi}\right)^{1 / 2}$ which gives the asymptotic behavior of the bare string coupling as

$$
g_{s}^{2}=e^{\phi} \simeq e^{c \varphi} .
$$

In view of the fact that, in the strong-coupling limit we are interested in, the factor $F(\varphi)$ in Eq. (2.4) quickly tends to a constant, we can simplify our analysis (without modifying the essential physics) by replacing it by a constant (which can then be absorbed in a redefinition of $\chi$ ). Henceforth, we shall simply take $F(\varphi)=1$. (See, however, the comments below concerning the self-regenerating inflationary regime.)

Following $[4,9]$ it is then useful to combine the Friedmann equations for the scale factor $a(t)$ during inflation $\left[d s^{2}=-d t^{2}+a^{2}(t) \delta_{i j} d x^{i} d x^{j}\right]$ with the equations of motion of the two scalar fields $\chi(t), \varphi(t)$, to write an autonomous equation describing the evolution of the two scalars in terms of the parameter 


$$
p=\int H d t=\int \frac{\dot{a}}{a} d t=\ln a+\text { const }
$$

measuring the number of $e$-folds of the expansion. For any multiplet of scalar fields, $\boldsymbol{\varphi}=\left(\varphi^{a}\right)$, this yields the simple equation $[4,9]$

$$
\frac{2}{3-\boldsymbol{\varphi}^{\prime 2}} \boldsymbol{\varphi}^{\prime \prime}+2 \boldsymbol{\varphi}^{\prime}=-\nabla_{\boldsymbol{\varphi}} \ln |V(\boldsymbol{\varphi})|,
$$

where $\boldsymbol{\varphi}^{\prime} \equiv d \boldsymbol{\varphi} / d p$, and where all operations on $\boldsymbol{\varphi}$ are covariantly defined in terms of the $\sigma$-model metric defining the scalar kinetic terms $\left[d \sigma^{2}=\gamma_{a b}(\varphi) d \varphi^{a} d \varphi^{b}\right]$. In our simple model [with $F(\varphi)=1$ ], we have a flat metric $d \sigma^{2}=d \varphi^{2}$ $+d \chi^{2}$. [Note that, when $\gamma_{a b}(\varphi)$ is curved the acceleration term $\varphi^{\prime \prime}$ involves a covariant derivative.]

The generic solution of Eq. (2.8) is easily grasped if one interprets it as a mechanical model: a particle with position $\boldsymbol{\varphi}$, and velocity-dependent mass $m\left(\boldsymbol{\varphi}^{\prime}\right)=2 /\left(3-\boldsymbol{\varphi}^{\prime 2}\right)$, moves, in the "time" $p=\ln a+c s t$, in the manifold $d \sigma^{2}$ under the influence of an external potential $\ln |V(\varphi)|$ and a constant friction force $-2 \varphi^{\prime}$. If the curvature of the effective potential $\ln |V(\boldsymbol{\varphi})|$ is sufficiently small the motion of $\boldsymbol{\varphi}$ rapidly becomes slow and friction dominated:

$$
2 \frac{d \boldsymbol{\varphi}}{d p} \simeq-\nabla_{\boldsymbol{\varphi}} \ln V(\boldsymbol{\varphi})
$$

Equation (2.9) is equivalent to the usual "slow roll" approximation.

Consistently with our general assumption (1.3), we consider potentials allowing a strong-coupling expansion of the form

$$
V(\chi, \varphi)=V_{0}(\chi)+V_{1}(\chi) e^{-c \varphi}+\mathcal{O}\left(e^{-2 c \varphi}\right),
$$

where $V_{0}(\chi)$ is a typical chaotic-inflation potential with $V_{0}(0)=0$, while $V_{1}(0)=v_{1} \geqslant 0$ can possibly provide (if $v_{1}$ $>0)$ the effective cosmological constant driving today's acceleration in the scenario of [6]. For the sake of simplicity we shall discuss mainly the "factorized" power-law case $V_{0}(\chi) \sim V_{1}(\chi) \sim \chi^{n}$ for which we can conveniently write $V$ in the form

$$
V(\chi, \varphi)=\lambda(\varphi) \frac{\chi^{n}}{n}
$$

with a dilaton-dependent coupling constant $\lambda(\varphi)$ of the form

$$
\lambda(\varphi)=\lambda_{\infty}\left(1+b_{\lambda} e^{-c \varphi}\right) .
$$

This example belongs to the class of the two-field inflationary potentials discussed in [10]. We have checked that our results remain qualitatively the same for the more general potential (2.10) provided that $V_{0}(\chi)$ and $V_{1}(\chi)$ are not extremely different and given the fact that $v_{1}$ is phenomenologically constrained to be very small. [Note that, within the simplified model (2.12), the ratio $V_{1}(\chi) / V_{0}(\chi)$ is equal to the constant coefficient $b_{\lambda}$.]
The universal (positive) constant $c$ appearing in the exponential $e^{-c \varphi}$ is the same as in Eq. (2.6) [i.e., $c$ $\equiv\left(2 C_{g} / C_{\phi}\right)^{1 / 2}$, which is expected to be of order unity]. The coefficient $b_{\lambda}$ in Eq. (2.12) is such that $b_{\lambda} e^{-c \varphi} \simeq b_{\lambda} e^{-\phi}$ roughly corresponds to a combination of terms $\sim \pm C_{i}^{-1} \mathcal{O}\left(e^{-\phi}\right)$ coming from the strong-coupling asymptotics of several $B_{i}(\phi)$, Eq. (1.3) [see Eq. (2.5)]. In the toy model of [5] one would therefore expect $b_{\lambda}$ to be smallish. Anyway, we shall see that in final results only the ratios of such $b_{i}$ coefficients enter. More important than the magnitude of $b_{\lambda}$ is its sign. It is crucial for the present strongcoupling attractor scenario to assume that $b_{\lambda}>0$, i.e., that $\lambda(\varphi)$ reaches a minimum at strong coupling, $\varphi \rightarrow+\infty$. Note again that this behavior is consistent with the simple "large $N$ "-type idea of [5] if we assimilate $\lambda(\varphi)$ into one of the inverse couplings $B_{i}$ appearing in Eq. (1.1) (for instance $B_{F} \sim g_{F}^{-2}$, where $g_{F}$ is a gauge coupling), rather than to the coupling itself. If the latter were the case, $\lambda(\varphi)$ would reach a maximum as $\phi \rightarrow+\infty$, and the attractor mechanism of [3] would drive $\phi$ toward weak coupling $(\phi \rightarrow-\infty)$. However, the Einstein-frame $\phi$ dependence of $V(\chi)$ gets contributions from several $B_{i}^{ \pm n}(\phi)$, Eq. (2.5), which might conspire to minimize it at strong coupling. This feature is also probably necessary in order to solve the cosmological-constant problem through some argument by which the vacuum at infinity has vanishing energy density.

Substituting the potential (2.10) into the slow roll equation (2.9) and assuming (for simplicity) that $V_{1}(\chi) e^{-c \varphi}$ is significantly smaller than $V_{0}(\chi)$ leads to a decoupled set of evolution equations for $\chi$ and $\varphi$ (where $\left.V^{\prime} \equiv \partial V / \partial \chi\right)$ :

$$
\begin{aligned}
& \frac{d \chi}{d p}=-\frac{1}{2} \frac{V_{0}^{\prime}}{V_{0}}, \\
& \frac{d \varphi}{d p}=\frac{1}{2} c e^{-c \varphi} \frac{V_{1}}{V_{0}} .
\end{aligned}
$$

Given some "initial" conditions $\chi_{\text {in }}, \varphi_{\text {in }}$ (discussed below) at some starting point, say $p=0$, the solution of Eqs. (2.13), (2.14) is simply

$$
\begin{aligned}
p & =2 \int_{\chi}^{\chi_{\text {in }}} d \bar{\chi} \bar{\chi}\left(\frac{V_{0}(\bar{\chi})}{\bar{\chi} V_{0}^{\prime}(\bar{\chi})}\right), \\
e^{c \varphi} & =e^{c \varphi_{\text {in }}}+\frac{c^{2}}{2} \int d p \frac{V_{1}(\chi(p))}{V_{0}(\chi(p))},
\end{aligned}
$$

which simply become

$$
\begin{gathered}
p=\frac{1}{n}\left(\chi_{\text {in }}^{2}-\chi^{2}\right), \\
e^{c \varphi}+\frac{b_{\lambda} c^{2}}{2 n} \chi^{2}=\text { const }=e^{c \varphi_{\text {in }}}+\frac{b_{\lambda} c^{2}}{2 n} \chi_{\text {in }}^{2},
\end{gathered}
$$

in the simplified case of Eqs. (2.11),(2.12).

Equations (2.17) show that, in order for the string coupling $g_{s}^{2} \simeq e^{c \varphi}$ to have reached large values at the end of 
inflation, a large total number of $e$-folds must have occurred while the (dimensionless) inflaton field $\chi$ decreased from a large initial value to a value of order unity (in Planck units). To get a quantitative estimate of the string coupling at the end of inflation we need to choose the initial conditions $\chi_{\text {in }}, \varphi_{\text {in }}$. A physically reasonable way (which is further discussed below) of choosing $\chi_{\text {in }}$ is to start the classical evolution (2.13)-(2.17) at the exit of the era of self-regenerating inflation (see [10] and references therein). We will now show how to relate the exit from self-regenerating inflation to the size of density fluctuations generated by inflation.

Let us recall (see [10] and references therein) that the density fluctuation $\delta \equiv \delta \rho / \rho$ on large scales (estimated in the one-field approximation where the inflaton $\chi$ is the main contributor) is obtained by evaluating the expression

$$
\delta(\chi) \simeq \frac{4}{3} \frac{1}{\pi}\left(\frac{2}{3}\right)^{1 / 2} \frac{V^{3 / 2}}{\partial_{\chi} V}
$$

at the value $\chi=\chi_{\times}$, at which the physical scale we are considering crossed the horizon outward during inflation. For the scale corresponding to our present horizon this usually corresponds to a value $\chi_{\times}\left(H_{0}\right)$ ( $\chi_{H}$ for short) reached some 60 $e$-folds before the end of slow roll. From Ref. [10], $\chi_{H}$ $\simeq 5 \sqrt{n}$ for the model (2.11) (and with our modified definition of $\chi$ ). The numerical value of $\delta_{H} \equiv \delta\left(\chi_{H}\right)$ which is compatible with cosmological data (structure formation and cosmic microwave background) is $\delta_{H} \simeq 5 \times 10^{-5}$. In the model (2.11) the function $\delta(\chi)$ defined by Eq. (2.18) scales with $\chi$ as $\chi^{(n+2) / 2}$. Putting together this information we obtain a relation between $\chi_{\text {in }}$ and $\delta\left(\chi_{\text {in }}\right)$, which involves the value of the observable horizon-size fluctuations $\delta_{H} \equiv \delta\left(\chi_{H}\right)$ :

$$
\frac{\delta\left(\chi_{\mathrm{in}}\right)}{\delta\left(\chi_{H}\right)}=\left(\frac{\chi_{\mathrm{in}}}{\chi_{H}}\right)^{(n+2) / 2}
$$

i.e.,

$$
\chi_{\mathrm{in}} \simeq \chi_{H}\left(\frac{\delta_{\text {in }}}{\delta_{H}}\right)^{2 /(n+2)} \simeq 5 \sqrt{n}\left(\frac{\delta_{\text {in }}}{\delta_{H}}\right)^{2 /(n+2)},
$$

where we introduced the shorthand notation $\delta_{\text {in }} \equiv \delta\left(\chi_{\text {in }}\right)$.

Inserting Eq. (2.20) into Eq. (2.16) we then obtain the following estimate of the string coupling constant after inflation as a function of $\varphi_{\text {in }}$ and $\delta\left(\chi_{\text {in }}\right)$ :

$$
\begin{aligned}
& e^{c \varphi_{\mathrm{end}}-}-e^{c \varphi_{\mathrm{in}}} \simeq \frac{c^{2}}{2}\left\langle V_{1} / V_{0}\right\rangle p \sim \frac{c^{2}}{2 n}\left\langle V_{1} / V_{0}\right\rangle \chi_{\mathrm{in}}^{2} \\
& \sim \frac{25 c^{2}}{2}\left\langle V_{1} / V_{0}\right\rangle\left(\frac{\delta_{\text {in }}}{\delta_{H}}\right)^{4 /(n+2)},
\end{aligned}
$$

where $\left\langle V_{1} / V_{0}\right\rangle$ denotes the average value of $V_{1} / V_{0}$ : $\left\langle V_{1} / V_{0}\right\rangle \equiv \int d p\left(V_{1} / V_{0}\right) / \int d p$ [note that this average ratio is equal to $b_{\lambda}$ in the simplified model (2.12)].

To get a quantitative estimate of $e^{c \varphi_{\text {end }}}$ we still need to estimate the value of $\delta\left(\chi_{\text {in }}\right)$ corresponding to the chosen "initial" value of the inflaton. As we will now check, taking for $\chi_{\text {in }}$ the value corresponding to the exit from self- regenerating inflation corresponds simply to taking $\delta\left(\chi_{\text {in }}\right)$ $\sim 1$. Indeed, let us first recall that, during inflation, each (canonically normalized) scalar field (of mass smaller than the expansion rate $H$ ) undergoes typical quantum fluctuations of order $H /(2 \pi)$, per Hubble time (see, e.g., [10]). This implies (for our dimensionless fields) that the value of $\chi$ at the exit from self-regeneration, say $\chi_{\mathrm{ex}}$, is characterized by $\hat{H}_{\text {ex }} /(2 \pi) \approx\left[\partial_{\chi} V /(2 V)\right]_{\text {ex }}$, where $\hat{H} \equiv H / \tilde{m}_{P}$ is the dimensionless Hubble expansion rate and where the right-hand side (RHS) is the classical change of $\chi$ per Hubble time [corresponding to the RHS of Eq. (2.13)]. Using Friedmann's equation (in the slow-roll approximation) $\hat{H}_{\mathrm{ex}}^{2}$ $\approx(2 / 3) V\left(\chi_{\text {ex }}\right)$, it is easily seen that that the exit from selfregeneration corresponds to $\delta\left(\chi_{\mathrm{ex}}\right) \approx 4 / 3 \sim 1$. It is, a posteriori, physically quite reasonable to start using the classical evolution system only when the (formal extrapolation) of the density fluctuation $\delta(\chi)$ becomes smaller than 1 .

Within some approximation (see [10]), one can implement the effect of the combined quantum fluctuations of $(\varphi, \chi)$ by adding random terms with rms values $\hat{H} / 2 \pi$ on the righthand side of Eqs. (2.13) and (2.14), $d \chi / d p$ and $d \varphi / d p$ being precisely the shifts of the fields in a Hubble time. The system of equations becomes thus of the Langevin type

$$
\begin{aligned}
& \frac{d \chi}{d p}=-\frac{1}{2} \frac{V_{0}^{\prime}}{V_{0}}+\frac{\hat{H}}{2 \pi} \xi_{1}, \\
& \frac{d \varphi}{d p}=\frac{1}{2} c e^{-c \varphi} \frac{V_{1}}{V_{0}}+\frac{\hat{H}}{2 \pi} \xi_{2},
\end{aligned}
$$

where $\hat{H} \approx[(2 / 3) V(\chi, \phi)]^{1 / 2}$ (in the slow-roll approximation) is the dimensionless expansion rate, and where $\xi_{1}$ and $\xi_{2}$ are (independent) normalized random white noises:

$$
\left\langle\xi_{i}\left(p_{1}\right) \xi_{j}\left(p_{2}\right)\right\rangle=\delta_{i j} \delta\left(p_{1}-p_{2}\right), \quad i, j=1,2 .
$$

When the random force terms dominate the evolution in either Eq. (2.22) or Eq. (2.23) the quasiclassical description (2.13),(2.14) breaks down. The phase space of the sytem can thus be roughly divided into four regions according to whether the evolution of none, one, or both of the two fields is dominated by quantum fluctuations. This is depicted in Fig. 1 where such regions are delimited by dashed, thick curves in the case of a power-law potential (2.11).

Apart from factors of order 1, the evolution of the inflaton $\chi$ is quasiclassical in the region under the line $\chi$ $=\lambda_{\infty}^{-1 /(n+2)}$. In the chaotic inflationary models [10] such an inflaton's value corresponds to the exit from the selfregenerating regime and to the beginning of the quasiclassical slow-roll inflation. As mentioned above it also corresponds to a perturbation $\delta(\chi) \sim 1$. Somewhat surprisingly, in the model at hand, however, the quasiclassical region for the inflaton evolution $\chi \leq \lambda_{\infty}^{-1 /(n+2)}$ is affected by quantum fluctuations that still dominate the evolution of $\varphi$ in the region above the hyperbolalike curve $\chi=b_{\lambda}^{2 / n} \lambda_{\infty}^{-1 / n} e^{-2 c \varphi / n}$. We must therefore study, in some detail, the evolution of the system in the presence of the noise term for $\varphi$ as in Eq. 


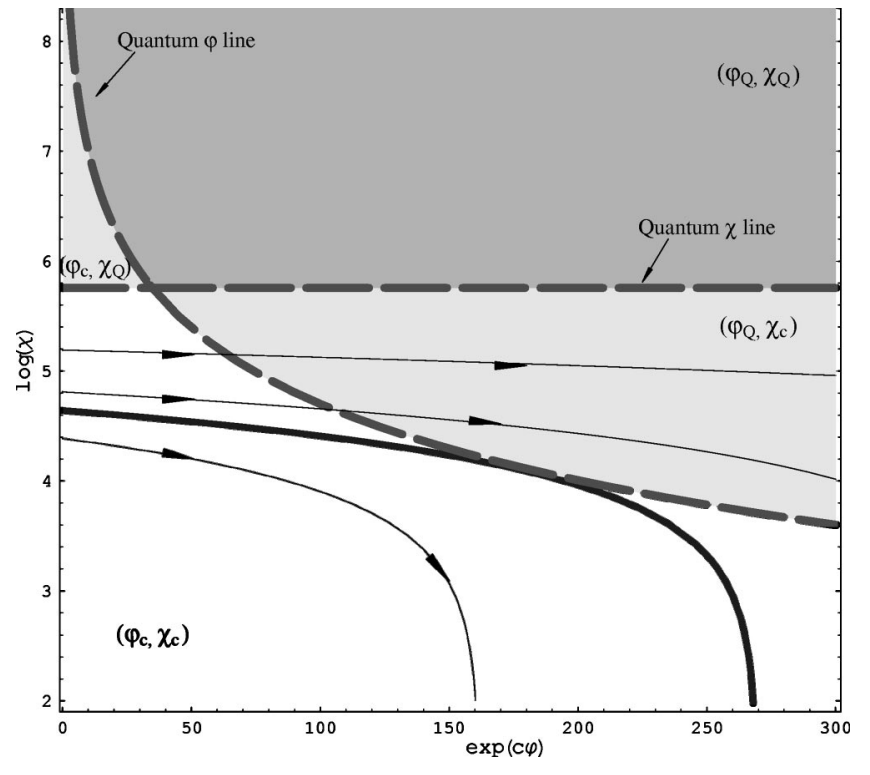

FIG. 1. The phase space of the system is represented in the case of a power-law potential (2.11) with $n=2, b_{\lambda}=0.1$, and $\lambda_{\infty}$ $=10^{-10}$. The thick-dashed curves delimit the quantum behavior of the two fields, the horizontal curve $\chi=\lambda_{\infty}^{-1 /(n+2)}$ and the hyperbolalike curve $\chi=b_{\lambda}^{2 / n} \lambda_{\infty}^{-1 / n} e^{-2 c \varphi / n}$ being the limits of the quantum behavior for $\chi$ and $\varphi$, respectively. In the white region both fields have a classical behavior. The last "fully classical" trajectory has been represented by a thick solid curve. The bright-gray regions are those where either the $\varphi$ or the $\chi$ evolution is dominated by quantum fluctuations. The fully quantum region is the dark-gray region on the top right.

(2.23), assuming a quasiclassical evolution for $\chi$. This is done in the Appendix. The final result is that, if we start at $\chi \lesssim \lambda_{\infty}^{-1 /(n+2)}$ (classical evolution for $\chi$ ), the average value of $e^{c \varphi}$ is multiplicatively renormalized, by a factor of order unity, with respect to the classical trajectory $e^{c \varphi_{\mathrm{cl}}}$, given by solving Eqs. (2.13), (2.14), i.e., $\left\langle e^{c \varphi}\right\rangle=\mathcal{O}(1) e^{c \varphi_{\mathrm{cl}}}$. One also finds that the dispersion of $e^{c \varphi}$ around its average value is comparable to its average value.

We shall not try to discuss here what happens in the selfregenerating region $\chi \gtrsim \chi_{\text {in }} \sim \lambda_{\infty}^{-1 /(n+2)}$. Let us recall that the simple decoupled system $(2.13),(2.14)$ was obtained by neglecting the kinetic coupling term $F(\varphi)$ in Eq. (2.4). If we were to consider a more general model, we would have more coupling between $\chi$ and $\varphi$ and we would expect that (contrary to Fig. 1 which exhibits a "classical $\varphi$ region" above the "quantum $\chi$ line") the evolution in the self-regenerating region would involve a strongly coupled system of Langevin equations. Then, as discussed in [10], solving such a system necessitates giving boundary conditions on all the boundaries of the problem: notably for $\chi \rightarrow \infty$, but also for $\varphi \rightarrow+\infty$ and $\varphi \rightarrow-\infty$. We leave to future work such an investigation (and a discussion of what are reasonable boundary conditions). In this work we shall content ourselves with "starting" the evolution on the quantum $\chi$ boundary line $\chi_{\text {in }}$ with some value $\varphi=\varphi_{\text {in }}$, assuming that $e^{c \varphi_{\text {in }}}$ is smaller than the driving effect due to inflation, i.e., than the RHS of Eq. (2.21). (This assumption is most natural in a work aimed at studying the "attracting" effect due to primordial inflation.)
Going back to our result (2.21), we can now insert, according to the preceding discussion, the values $\delta_{\text {in }}=1$ and $e^{c \varphi_{\text {in }} \ll} e^{c \varphi_{\text {end }}}$. Finally, in the simplified model (2.11),(2.12), we get the estimate

$$
\begin{aligned}
e^{c \varphi_{\mathrm{end}}} & =\mathcal{O}(1) \cdot e^{c \varphi_{\mathrm{cl}, \text { end }}} \\
& \sim \mathcal{O}(1) \cdot \frac{25 c^{2}}{2} b_{\lambda}\left(\delta_{H}\right)^{-4 /(n+2)} .
\end{aligned}
$$

A more general analysis based on the potential (2.10) leads to the same final result but with $n$ replaced by some average value of $\chi V_{0, \chi} / V_{0}$, and with $b_{\lambda}$ replaced by some average of the ratio $V_{1} / V_{0}$. Note that smaller values of the exponent $n$ lead to larger values of $e^{c \varphi_{\text {end }}}$, i.e., to a more effective attraction toward the "fixed point at infinity." The same is true if we take different exponents $n_{0}$ and $n_{1}$ (for $V_{0}$ and $V_{1}$, respectively) and assume $V_{1}\left(\chi_{\text {in }}\right) \gg V_{0}\left(\chi_{\text {in }}\right)$ to hold as a result of $\chi_{\text {in }} \gg 1$ and $n_{1}>n_{0}$. Also note that, numerically, if we consider $n=2$, i.e., the simplest chaotic-inflation potential $V=\frac{1}{2} m_{\chi}^{2}(\varphi) \chi^{2}$, Eq. (2.25) involves the large number $12.5 \times \delta_{H}^{-1} \sim 2.5 \times 10^{5}$. In the case where $n=4$, i.e., $V$ $=\frac{1}{4} \lambda(\varphi) \chi^{4}$, we have instead the number $12.5 \times \delta_{H}^{-2 / 3}$ $\sim 0.92 \times 10^{4}$. To understand the phenomenological meaning of these numbers we need to relate $e^{c \varphi_{\text {end }}}$ to the present, observable deviations from general relativity. This issue is addressed in Sec. III after having argued that the postinflationary evolution of $\varphi$ is subdominant.

\section{B. Attraction of $\varphi$ by the subsequent cosmological evolution}

We have discussed above the efficiency with which inflation drives the dilaton toward a fixed point at infinity. We need to complete this discussion by estimating the effect of the many $e$-folds of expansion that took place between the end of inflation and the present time. To address this question, we need to study in more detail the coupling of a runaway dilaton to various types of matter, say, a multicomponent distribution of (relativistic or nonrelativistic) particles. We work in the Einstein frame, with an action of the type

$$
\begin{aligned}
S= & \int d^{4} x \sqrt{g}\left[\frac{\tilde{m}_{P}^{2}}{4} R-\frac{\tilde{m}_{P}^{2}}{2}(\nabla \varphi)^{2}-\frac{1}{4} B_{F}(\varphi) F^{2}+\cdots\right] \\
& -\sum_{A} \int m_{A}\left[\varphi\left(x_{A}\right)\right] \sqrt{-g_{\mu \nu}\left(x_{A}\right) d x_{A}^{\mu} d x_{A}^{\nu}} .
\end{aligned}
$$

Following $[2,3]$, one introduces the crucial dimensionless quantity

$$
\alpha_{A}(\varphi) \equiv \frac{\partial \ln m_{A}(\varphi)}{\partial \varphi}
$$

measuring the coupling of $\varphi$ to a particle of type $A$. (For consistency with previous work, we keep the notation $\alpha_{A}$ but warn the reader that this should not be confused with the 
various gauge coupling constants, often denoted $\alpha_{i}$ $=g_{i}^{2} / 4 \pi$.) The quantity $\alpha_{A}$ determines the effect of cosmological matter on the evolution of $\varphi$ through the general equation $[4,3]$

$$
\frac{2}{3-\varphi^{\prime 2}} \varphi^{\prime \prime}+\left(1-\frac{P}{\rho}\right) \varphi^{\prime}=-\sum_{A} \alpha_{A}(\varphi) \frac{\rho_{A}-3 P_{A}}{\rho},
$$

where the primes denote derivatives with respect to $p=\ln a$ + const and where $\rho=\Sigma_{A} \rho_{A}$ and $P=\Sigma_{A} P_{A}$ are the total "material" energy density and pressure, respectively, both obtained as sums over the various components filling the universe with the exception of the kinetic energy density and pressure of $\varphi, \rho_{k}=\left(\tilde{m}_{P}^{2} / 2\right)(d \varphi / d t)^{2}=\left(\tilde{m}_{P}^{2} / 2\right) H^{2} \varphi^{\prime 2}$ and $P_{k}$ $=\rho_{k}$. Accordingly, the Friedmann equation reads

$$
3 H^{2}=\frac{2}{\tilde{m}_{P}^{2}} \rho_{\mathrm{tot}}=\frac{2 \rho}{\tilde{m}_{P}^{2}}+H^{2} \varphi^{\prime 2} .
$$

Note that $\rho$ and $P$ may also account for the potential energy density and pressure of the scalar field, $\rho_{V}=V(\varphi)$, $P_{V}=-\rho_{V}$, and that one can formally extend Eq. (2.28) to the "vacuum energy" component $V(\varphi)$ by associating with the potential $V(\varphi)$ the mass scale $m_{V}(\varphi) \equiv V(\varphi)^{1 / 4}$ which gives $\alpha_{V}=\partial \ln m_{V}(\varphi) / \partial \varphi=\frac{1}{4} \partial \ln V(\varphi) / \partial \varphi$. Equation (2.8) is then recovered in the limit where the scalar field is the dominant component.

In the simple cases (which are quite frequent, at least as approximate cases) where one "matter" component, with known "equation of state" $P_{A} / \rho_{A}=w_{A}=$ const, dominates the cosmological density and pressure, Eq. (2.28) yields an autonomous equation for the evolution (with redshift) of $\varphi$. Using Eq. (2.29) one finds that the "equation of state parameter" $w_{\text {tot }} \equiv P_{\text {tot }} / \rho_{\text {tot }}$ corresponding to the total energy and pressure [including now the kinetic contributions of $\varphi$; i.e., $\left.\rho_{\text {tot }}=\rho+\left(\tilde{m}_{P}^{2} / 2\right)(d \varphi / d t)^{2}, P_{\text {tot }}=P+\left(\tilde{m}_{P}^{2} / 2\right)(d \varphi / d t)^{2}\right] \quad$ is given in terms of the "matter" equation-of-state parameter $w \equiv P / \rho$ by

$$
w_{\text {tot }}=w+\frac{1-w}{3}\left(\varphi^{\prime}\right)^{2} .
$$

The knowledge of $w_{\text {tot }}$ then allows one to write explicitly the energy-balance equation $d \rho_{\text {tot }}+3\left(\rho_{\text {tot }}+P_{\text {tot }}\right) d \ln a=0$, which is easily solved in the simple cases where $w_{\text {tot }}$ is (approximately) constant.

We see from Eq. (2.28) that, during the radiation era (starting, say, immediately after the end of inflation), i.e., when the universe is dominated by an ultrarelativistic gas $\left(\rho_{A}-3 P_{A}=0\right)$, the "driving force" on the right-hand side of Eq. (2.28) vanishes, so that $\varphi$ is not driven further away toward infinity. Actually, one should take into account both the "inertial" effect of the "velocity" $\varphi$ ' acquired during the preceding inflationary driving of $\varphi$, and the integrated effect of the many "mass thresholds" $T_{A} \sim m_{A}$, when some component becomes nonrelativistic (so that $\rho_{A}-3 P_{A} \neq 0$ ). Using the results of $[4,3]$ one sees that, in our case, both these effects have only a small impact on the value of $\varphi$. Therefore, to a good approximation $\varphi \simeq \varphi_{\text {end }}$ until the end of the radiation era.

On the other hand, when the universe gets dominated by nonrelativistic matter, one gets a nonzero driving force in Eq. (2.28). In the slow-roll approximation, as the transient behavior has died out, since $w=P / \rho$ becomes negligible, we have simply

$$
\varphi_{m}^{\prime}=-\alpha_{m}(\varphi)
$$

where $\varphi_{m}^{\prime}$ stands for the $\varphi$ velocity during matter domination; and $\alpha_{m}(\varphi)$ denotes the coupling (2.27) to dark matter.

The coupling to dark matter, $\alpha_{m}(\varphi)$, depends on the assumption one makes about the asymptotic behavior, at strong bare string coupling, of the mass of the weakly interacting massive particles (WIMPs) constituting the dark matter. One natural looking, minimal assumption is that dark matter, like all visible types of matter, is coupled in a way which levels off at strong bare coupling, as in Eq. (1.3). In other words, one generally expects that $m_{m}(\varphi) \simeq m_{m}(+\infty)\left(1+b_{m} e^{-c \varphi}\right)$ so that $\alpha_{m}(\varphi) \simeq-b_{m} c e^{-c \varphi}$. It is then easy to solve Eq. (2.31), with initial conditions $\varphi_{0}=\varphi_{\text {end }}, \varphi_{0}^{\prime}=0$ (inherited from the radiation era) at the beginning of the matter era. But we shall not bother to write the explicit solution because it is easily seen that the smallness of $e^{-c \varphi}$ end guarantees that the "driving force" $\propto \alpha_{m}(\varphi)$ always remains so small that the $\mathcal{O}(10) e$-folds of the matter era until vacuum-energy domination (or until the present) have only a fractionally negligible effect on $\varphi$.

A more significant evolution of $\varphi$ during the matter era is provided if, as first proposed in [11] and taken up in [12,13], dark matter couples much more strongly to $\varphi$ than "ordinary" matter. Such a stronger coupling to dark matter, which is not constrained by the usual equivalence principle experiments, follows from assuming more general quantum corrections in the dark matter sector of the theory, i.e., corrections such that the dark matter mass $m_{m}(\varphi)$, instead of levelling off, either vanishes or keeps increasing at strong bare coupling: $m_{m}(\varphi) \propto e^{c_{m} \varphi}$, so that $\alpha_{m}=c_{m}$ is a (negative or positive) constant. In [6] (but see also [14]) it has been shown that under the latter assumption (i.e., with a positive coupling parameter $\alpha_{m}>0$ ) the dilaton can play the role of quintessence, leading to a late-time cosmology of accelerated expansion. By Eq. (2.31) we have $\varphi=\varphi_{\text {end }}-\alpha_{m} p$, where $p$ is now counted from the end of the radiation era. Given that about nine e-folds separate us from the end of the radiation era, we see that such an evolution might (if $\left|\alpha_{m}\right|$ is really of order unity) have a significant effect on the present value of $\varphi$ [when compared with the value at the end of inflation, i.e., $\left.c \varphi_{\text {end }} \sim \ln \left(1 / \delta_{H}\right) \sim 10\right]$. However, the running of $\varphi$ during the matter era changes the standard recent cosmological picture and is therefore constrained by observations. In fact, by Eq. (2.30), the total matter-era equation of state parameter $w_{\text {tot }}$ in the presence of the dilaton reads $w_{\text {tot }}=\left(\varphi_{m}^{\prime}\right)^{2} / 3$. Accordingly, the matter density varies as $\rho \propto a^{-3\left(1+w_{\text {tot }}\right)}=a^{-\left(3+\left(\varphi_{m}^{\prime}\right)^{2}\right)}$, possibly affecting the standard scenario of structure formation as well as the global temporal picture between now and 
the epoch of matter-radiation equality. The compatibility with phenomenology therefore puts constraints on the magnitude of $\varphi_{m}^{\prime 2}=\alpha_{m}(\varphi)^{2}$. In [6] $w_{\text {tot }}<0.1$, i.e., $v \equiv \varphi_{m}^{\prime 2} / 0.3$ $<1$, was suggested to be the maximal deviation one can roughly tolerate during the matter era, the establishment of a more precise bound being presently under study [15]. We shall therefore assume either that we are in the "normal" case where the dilaton does not couple more strongly to dark matter than to ordinary matter [so that $\alpha_{m}(\varphi) \simeq-b_{m} c e^{-c \varphi}$ $\ll 1]$, or that, if it does, $\alpha_{m}^{2}<0.3$. This leads to a displacement of $\varphi$ during the matter era smaller than the dispersion $\varphi_{\mathrm{end}}-\varphi_{\mathrm{cl} \text {, end }} \sim \varphi_{\mathrm{cl} \text {, end }}$ produced by quantum fluctuations during inflation, Eq. (2.25).

In this context one should also consider the attraction effect of a negative pressure component, either in the form of a $\varphi$-dependent vacuum energy (dilatonic quintessence) or in the form of any other, $\varphi$-independent component (such as a "genuine" cosmological constant). Of course, the present recent $(z \lesssim 1)$ accelerated expansion phase is very short (in " $p$ time") and sensible changes of the dilaton value since the end of matter domination are not expected. Still, it is crucial to estimate the present dilaton velocity $\varphi_{0}^{\prime}$ since it is related to the cosmological variations of the coupling constants (see the next section). In the general case where both nonrelativistic matter and (possibly $\varphi$-dependent) vacuum energy density $V(\varphi)$ are present, the value of $\varphi_{0}^{\prime}$ predicted by our model is obtained by applying Eq. (2.28) (in the slow-roll approximation):

$$
\begin{aligned}
\left(\Omega_{m}+\Omega_{V}\right)\left(1-w_{0}\right) \varphi_{0}^{\prime} & =\left(\Omega_{m}+2 \Omega_{V}\right) \varphi_{0}^{\prime} \\
& =-\Omega_{m} \alpha_{m}-4 \Omega_{V} \alpha_{V} .
\end{aligned}
$$

In the above expression $\Omega_{m}$ and $\Omega_{V}$ are, respectively, the nonrelativistic (dark) matter and the vacuum fraction of the critical energy density $\left[\rho_{c} \equiv(3 / 2) \tilde{m}_{P}^{2} H^{2}\right]$, and the already mentioned prescriptions $\alpha_{V}=\frac{1}{4} \partial \ln V(\varphi) / \partial \varphi, \quad P_{V}=-\rho_{V}$ $=-V(\varphi)$ have been used.

The value of $\varphi_{0}^{\prime}$ is therefore some combination of the values of $\alpha_{m}$ and $\alpha_{V}$. We can have two classes of contrasting situations: In the first class, the dilaton couples "normally" (i.e., weakly) both to dark matter and to dark energy, i.e., both $\alpha_{m} \simeq-b_{m} c e^{-c \varphi} \ll 1$ and $\alpha_{V} \ll 1$ and Eq. (2.32) implies $\varphi_{0}^{\prime} \ll 1$. In the second class, the dilaton couples more strongly to some type of dark matter or energy, i.e., either (or both) $\alpha_{m}$ or/and $\alpha_{V}$ is of order unity so that $\varphi_{0}^{\prime}=\mathcal{O}(1)$. The second case is realized in the scenario of [6]. In the context of this scenario we have an exponential dependence of the potential on $\varphi, V(\varphi) \simeq V_{1} e^{-c \varphi}$ so that $\alpha_{V} \simeq-(c / 4)$ and

$$
\varphi_{0}^{\prime}=\frac{c \Omega_{V}-\alpha_{m} \Omega_{m}}{2 \Omega_{V}+\Omega_{m}} \lesssim \frac{c}{3} .
$$

The last inequality follows from the bound $\alpha_{m}>c / 2$ (which is a necessary condition to have positive acceleration in the model [6]) and the reasonable bound $\Omega_{m}>0.25$.

In the present work, we wish, however, to be as independent as possible from specific assumptions (such as the ones used in [6]). Therefore, rather than insisting on specific (model-dependent) predictions for the present value of $\varphi_{0}^{\prime}$ we wish to find the (model-independent) upper bounds on the possible values of $\varphi_{0}^{\prime}$ set by current observational data. There are several ways of getting such phenomenological bounds, because the existence of a kinetic energy (and pressure) associated with $d \varphi / d t=H \varphi^{\prime}$ has several observable consequences. A rather secure bound can be obtained by relating the value of $\varphi^{\prime}$ to the deceleration parameter $q \equiv-\ddot{a} a / \dot{a}^{2}$. In the general class of models that we consider, the cosmological energy density and pressure have (currently) three significant contributions: dark matter $\left(\Omega_{m}=\rho_{m} / \rho_{c}\right)$, dark energy $\left(\Omega_{V}\right)$, and the kinetic effect of a scalar field $\left[\Omega_{k}=\rho_{k} / \rho_{c}\right.$ with $\rho_{k}=\left(\tilde{m}_{P}^{2} / 2\right)(d \varphi / d t)^{2}=\left(\tilde{m}_{P}^{2} / 2\right) H^{2} \varphi^{\prime 2} \quad$ so that $\Omega_{k}$ $\left.=\varphi^{\prime 2} / 3\right]$. We assume (consistently with recent cosmic background data) that the space curvature is zero. Therefore we have the first relation

$$
\Omega_{m}+\Omega_{V}+\Omega_{k}=1=\Omega_{m}+\Omega_{V}+\varphi^{\prime 2} / 3 .
$$

The deceleration parameter is given by the general expression $2 q=\Sigma_{A} \Omega_{A}\left(1+3 w_{A}\right)$. Using $w_{m}=0, w_{V}=-1$, and $w_{k}=+1$, we get

$$
2 q=\Omega_{m}-2 \Omega_{V}+\frac{4}{3} \varphi^{\prime 2} .
$$

Using the relation (2.34) above to eliminate $\Omega_{V}$ we get the following expression for $\varphi^{\prime 2}$ in terms of the observable quantities $q$ and $\Omega_{m}$ :

$$
\varphi^{\prime 2}=1+q-\frac{3}{2} \Omega_{m}
$$

The supernovae Ia data [16] give a strict upper bound on the present value $q_{0}: q_{0}<0$. A generous lower bound on the present value of $\Omega_{m}$ is $\Omega_{m 0}>0.2$ [17]. Inserting these two constraints in Eq. (2.36) finally yields the safe upper bound

$$
\varphi_{0}^{\prime 2}<0.7 \text {, i.e., }\left|\varphi_{0}^{\prime}\right|<0.84 \text {. }
$$

To summarize, quite different rates of evolution for the dilaton are possible. A very slow variation is expected whenever dilaton couplings to both dark energy and dark matter follow the "normal" behavior (1.3). Otherwise, dilaton variations on the Hubble scale are expected. However, cosmological observations set the strict upper bound (2.37) on the present time variation of $\varphi$. For the purpose of the present section (evaluating the current location of the dilaton) these two alternatives do not make much difference because the vacuum-dominance era started less than about 0.7 $e$-folds away $\left[\ln \left(1+z_{*}\right)\right.$ with $\left.z_{*}<1\right]$. Therefore, $\varphi$ did not have enough " $p$ time," during vacuum dominance, to move much, even if it is coupled to vacuum energy with $\alpha_{V} \simeq$ $-(c / 4) \sim 1$.

Finally, we conclude from this analysis that, to a good approximation (and using the fact that the phenomenology of the matter era constrains the dark-matter couplings of the 
dilaton to be rather small), the value of $\varphi$ now is essentially given by the value $\varphi_{\text {end }}$ at the end of inflation, i.e., by Eq. (2.21).

\section{DEVIATIONS FROM GENERAL RELATIVITY INDUCED BY A RUNAWAY DILATON}

\section{A. Composition-independent deviations from general relativity}

The previous section reached the conclusion that present deviations from general relativity are given, to a good approximation, by the values of the matter-coupling coefficients $\alpha_{A}(\varphi)$ given by Eq. (2.27) calculated at $\varphi \simeq \varphi_{\text {end }}$ as given by Eq. (2.25). Let us now see the meaning of this result in terms of observable quantities.

Let us first consider the (approximately) compositionindependent deviations from general relativity, i.e., those that do not essentially depend on violations of the equivalence principle. Most composition-independent gravitational experiments (in the solar system or in binary pulsars) consider the long-range interaction between objects whose masses are essentially baryonic (the Sun, planets, neutron stars). As argued in $[2,3]$ the relevant coupling coefficient $\alpha_{A}$ is then approximately universal and given by the logarithmic derivative of the QCD confinement scale $\Lambda_{\mathrm{QCD}}(\varphi)$, because the mass of hadrons is essentially given by a pure number times $\Lambda_{\mathrm{OCD}}(\varphi)$. [We shall consider below the small, nonuniversal, corrections to $m_{A}(\varphi)$ and $\alpha_{A}(\varphi)$ linked to QED effects and quark masses.] Remembering from Eq. (1.1) the fact that, in the string frame [where there is a fixed cutoff linked to the string mass $\left.\tilde{M}_{s} \sim\left(\alpha^{\prime}\right)^{-1 / 2}\right]$ the gauge coupling is dilaton dependent $\left[g_{F}^{-2}=B_{F}(\varphi)\right]$, we see that (after conformal transformation) the Einstein-frame confinement scale has a dilaton dependence of the form

$$
\Lambda_{\mathrm{QCD}}(\varphi) \sim C^{-1 / 2} B_{g}^{-1 / 2}(\varphi) \exp \left[-8 \pi^{2} b_{3}^{-1} B_{F}(\varphi)\right] \tilde{M}_{s},
$$

where $b_{3}$ denotes the one-loop (rational) coefficient entering the renormalization group running of $g_{F}$. Here $B_{F}(\varphi)$ denotes the coupling to the SU(3) gauge fields. For simplicity, we shall assume that (modulo rational coefficients) all gauge fields couple (near the string cutoff) to the same $B_{F}(\varphi)$. This yields the following approximately universal dilaton coupling to hadronic matter:

$$
\alpha_{\text {had }}(\varphi) \simeq\left[\ln \left(\frac{\tilde{M}_{s}}{\Lambda_{\mathrm{QCD}}}\right)+\frac{1}{2}\right] \frac{\partial \ln B_{F}^{-1}(\varphi)}{\partial \varphi} .
$$

We recall that the quantity $\alpha_{\text {had }}(\varphi)$, which measures the coupling of the dilaton to hadronic matter, should not be confused with any "strong" gauge coupling, $\alpha_{s}=g_{s}^{2} / 4 \pi$. Numerically, the coefficient in front of the RHS of Eq. (3.2) is of order 40. Consistently with our basic assumption (1.3), we parametrize the $\varphi$ dependence of the gauge coupling $g_{F}^{2}$ $=B_{F}^{-1}$ as

$$
B_{F}^{-1}(\varphi)=B_{F}^{-1}(+\infty)\left[1-b_{F} e^{-c \varphi}\right]
$$

Note that, like $b_{\lambda}$ (see Sec. II A), $b_{F}$ is also expected to be smallish $\left[\sim B_{F}^{-1}(+\infty)\right.$ or, equivalently, $\sim C_{F}^{-1}$ in the notation of Eq. (1.3)] and typically the ratio $b_{F} / b_{\lambda}$ is of order unity. We finally obtain

$$
\alpha_{\text {had }}(\varphi) \simeq 40 b_{F} c e^{-c \varphi} .
$$

We can now insert the estimate (2.25) of the value of $\varphi$ reached because of the cosmological evolution. Neglecting the $\mathcal{O}(1)$ renormalization factor due to quantum noise, we get the estimate

$$
\begin{gathered}
\alpha_{\text {had }}\left(\varphi_{\text {end }}\right) \simeq 3.2 \frac{b_{F}}{b_{\lambda} c} \delta_{H}^{4 /(n+2)}, \\
\alpha_{\text {had }}^{2}\left(\varphi_{\text {end }}\right) \simeq 10\left(\frac{b_{F}}{b_{\lambda} c}\right)^{2} \delta_{H}^{8 /(n+2)} .
\end{gathered}
$$

As said above, it is plausible to expect that the quantity $c$ (which is a ratio) and the ratio $b_{F} / b_{\lambda}$ are both of order unity. This then leads to the numerical estimate $\alpha_{\text {had }}^{2} \sim 10 \delta_{H}^{8 /(n+2)}$, with $\delta_{H} \simeq 5 \times 10^{-5}$. An interesting aspect of this result is that the expected present value of $\alpha_{\text {had }}^{2}$ depends rather strongly on the value of the exponent $n$ [which entered the inflaton potential $V(\chi) \propto \chi^{n}$ ]. In the case $n=2$ [i.e., $V(\chi)=\frac{1}{2} m_{\chi}^{2} \chi^{2}$ ] we have $\alpha_{\text {had }}^{2} \sim 2.5 \times 10^{-8}$, while if $n=4\left[V(\chi)=\frac{1}{4} \lambda \chi^{4}\right]$ we have $\alpha_{\text {had }}^{2} \sim 1.8 \times 10^{-5}$.

How do these numbers compare to present (compositionindependent) experimental limits on deviations from Einstein's theory [18]? This question has been addressed in the literature. Concerning solar-system (post-Newtonian) tests it was shown (see, e.g., [19]) that the two main "Eddington" parameters $\gamma-1$ and $\beta-1$ measuring post-Newtonian deviations from general relativity are linked as follows to the dilaton coupling $\alpha_{\text {had }}(\varphi)$ :

$$
\begin{aligned}
& \gamma-1=-2 \frac{\alpha_{\text {had }}^{2}}{1+\alpha_{\text {had }}^{2}} \simeq-2 \alpha_{\text {had }}^{2}, \\
& \beta-1=\frac{1}{2} \frac{\alpha_{\text {had }}^{\prime} \alpha_{\text {had }}^{2}}{\left(1+\alpha_{\text {had }}^{2}\right)^{2}} \simeq \frac{1}{2} \alpha_{\text {had }}^{\prime} \alpha_{\text {had }}^{2},
\end{aligned}
$$

where $\alpha_{\text {had }}^{\prime} \equiv \partial \alpha_{\text {had }}(\varphi) / \partial \varphi$.

From Eq. (3.4) we see that $\alpha_{\text {had }}^{\prime} \simeq-c \alpha_{\text {had }}$, so that the deviation $\beta-1$ is $\mathcal{O}\left(\alpha_{\text {had }}^{3}\right)$ and thereby predicted to be too small to be phenomenologically interesting. This leaves $\gamma$ $-1 \simeq-2 \alpha_{\text {had }}^{2}$ as the leading observable deviation. The best current solar-system limit on $\gamma-1$ comes from very long baseline interferometry measurements of the deflection of radio waves by the Sun and is (approximately) $|\gamma-1|$ $\lesssim 2 \times 10^{-4}$, corresponding to $\alpha_{\text {had }}^{2} \leq 10^{-4}$ (see [18] for reviews and references). In addition to solar-system tests, we should also consider binary-pulsar tests which provide another high-precision window on possible deviations from general relativity. They have been analyzed in terms of the 
two quantities $\alpha_{\text {had }}$ (denoted $\alpha$ ) and $\alpha_{\text {had }}^{\prime}$ (denoted $\beta$ ) in [20]. The final conclusion is that the binary-pulsar limit on $\alpha_{\text {had }}$ is of order $\alpha_{\text {had }}^{2} \lesssim 10^{-3}$.

At this stage it seems that the runaway scenario explored here is leading to deviations from general relativity which are much smaller than present experimental limits. However, we must turn our attention to composition-dependent effects which turn out to be much more sensitive tests.

\section{B. Composition-dependent deviations from general relativity}

Let us then consider situations where the nonuniversal couplings of the dilaton induce (apparent) violations of the equivalence principle. Let us start by considering the composition dependence of the dilaton coupling $\alpha_{A}$, Eq. (2.27), i.e., the dependence of $\alpha_{A}$ on the type of matter we consider. The definition of $\alpha_{A}$ is such that, the Newtonian approximation, the interaction potential between particle $A$ and particle $B$ is $-G_{A B} m_{A} m_{B} / r_{A B}$ where [3]

$$
G_{A B}=G\left(1+\alpha_{A} \alpha_{B}\right) .
$$

Here, $G$ is the bare gravitational coupling constant entering the Einstein-frame action (2.4), and $\alpha_{A}=\alpha_{A}(\varphi)$ is the strength of the dilaton coupling to $A$ particles, taken at the present (cosmologically determined) VEV of $\varphi$. The term $\alpha_{A} \alpha_{B}$ comes from the additional attractive effect of dilaton exchange. Two test masses, made respectively of $A$ - and $B$-type particles, will then fall in the gravitational field generated by an external mass $m_{E}$ with accelerations differing by

$$
\left(\frac{\Delta a}{a}\right)_{A B} \equiv 2 \frac{a_{A}-a_{B}}{a_{A}+a_{B}} \simeq\left(\alpha_{A}-\alpha_{B}\right) \alpha_{E}
$$

We have seen above that in lowest approximation $\alpha_{A} \simeq \alpha_{\text {had }}$ does not depend on the composition of $A$. We need now, however, to retain the small composition-dependent effects in $\alpha_{A}$ linked to the $\varphi$ dependence of QED and quark contributions to $m_{A}$. This has been investigated in [3] with the result

$$
\left(\frac{\Delta a}{a}\right)_{A B}=\left(\frac{\alpha_{\mathrm{had}}}{40}\right)^{2}\left[C_{B} \Delta\left(\frac{B}{M}\right)+C_{D} \Delta\left(\frac{D}{M}\right)+C_{E} \Delta\left(\frac{E}{M}\right)\right]_{A B},
$$

where $(\Delta X)_{A B} \equiv X_{A}-X_{B}, B \equiv N+Z$ is the baryon number, $D \equiv N-Z$ the neutron excess, $E \equiv Z(Z-1) /(N+Z)^{1 / 3}$ a quantity linked to nuclear Coulomb effects, and $M \equiv m / u$ denotes the mass in atomic mass units, $u$ $=931.49432 \mathrm{MeV}$. It is difficult (and model dependent) to try to estimate the coefficients $C_{B}$ and $C_{D}$. It was argued in [3] that their contributions to Eq. (3.11) are generically expected to be subdominant with respect to the last contribution, proportional to $C_{E}$, which can be better estimated because it is linked to the $\varphi$ dependence of the fine-structure constant $e^{2} \propto B_{F}^{-1}(\varphi)$. This then leads to the numerical estimate $C_{E} \simeq 3.14 \times 10^{-2}$ and a violation of the universality of free fall approximately given by

$$
\left(\frac{\Delta a}{a}\right)_{A B} \simeq 2 \times 10^{-5} \alpha_{\mathrm{had}}^{2}\left[\left(\frac{E}{M}\right)_{A}-\left(\frac{E}{M}\right)_{B}\right]
$$

The values of $B / M, D / M$, and $E / M$ were computed in [21]. For mass pairs that have actually been used in recent experiments (such as beryllium and copper), as well as for mass pairs that are planned to be used in forthcoming experiments (such as platinum and titanium) one finds $(E / M)_{\mathrm{Cu}}$ $-(E / M)_{\mathrm{Be}}=2.56,(E / M)_{\mathrm{Pt}}-(E / M)_{\mathrm{Ti}}=2.65$. Using the average estimate $\Delta(E / M) \simeq 2.6$, we get from Eqs. (3.12) and (3.6) the estimate

$$
\begin{aligned}
\left(\frac{\Delta a}{a}\right) & \simeq 5.2 \times 10^{-5} \alpha_{\mathrm{had}}^{2} \\
& \simeq 5.2 \times 10^{-4}\left(\frac{b_{F}}{b_{\lambda} c}\right)^{2} \delta_{H}^{8 /(n+2)} .
\end{aligned}
$$

Note also [from Eq. (3.7)] the link between compositiondependent effects and post-Newtonian ones

$$
\left(\frac{\Delta a}{a}\right) \simeq-2.6 \times 10^{-5}(\gamma-1)
$$

As current tests of the universality of free fall (UFF) have put limits in the $10^{-12}$ range [e.g., $(\Delta a / a)_{\mathrm{Be} \mathrm{Cu}}=(-1.9$ $\pm 2.5) \times 10^{-12}$ from [22]], we see from Eq. (3.14) that this corresponds to limits on $\gamma-1$ or $\alpha_{\text {had }}^{2}$ in the $10^{-7}$ range. Therefore tests of the UFF put much more stringent limits on dilaton models than solar-system or binary-pulsar tests.

If we insert the estimate $\delta_{H} \sim 5 \times 10^{-5}$ in Eq. (3.13) we obtain a level of violation of UFF due to a runaway dilaton which is

$$
\begin{aligned}
& \frac{\Delta a}{a} \simeq 1.3\left(\frac{b_{F}}{b_{\lambda} c}\right)^{2} \times 10^{-12} \text { for } n=2, \\
& \frac{\Delta a}{a} \simeq 0.98\left(\frac{b_{F}}{b_{\lambda} c}\right)^{2} \times 10^{-9} \text { for } n=4 .
\end{aligned}
$$

At face value, one is tempted to conclude that a scenario with $n=4$ [i.e., $V(\chi) \propto \chi^{4}$ ] tends to be too weak an attractor toward $\varphi=+\infty$ to be naturally compatible with equivalenceprinciple tests. (See, however, the discussion below.) On the other hand, the simple scenario $n=2\left[V(\chi)=\frac{1}{2} m_{\chi}^{2} \chi^{2}\right]$ is quite appealing in that it naturally provides enough attraction toward $\varphi=+\infty$ to be compatible with all existing experimental tests. At the same time it suggests that a modest improvement in the precision of UFF experiments might discover a violation caused by a runaway dilaton.

\section{Cosmological variation of "constants"}

Let us now consider another possible deviation from general relativity and the standard model: a possible variation of the coupling constants, most notably of the fine-structure 
constant $e^{2} / \hbar c$ on which the strongest limits are available. We will discuss first the effects due to the cosmological time variation of the homogeneous component of $\varphi$ and, in the next subsection, the possible spatial (and time) variations due to quantum fluctuations of $\varphi$ as they were amplified during inflation.

Consistently with our previous assumptions we expect $e^{2} \propto B_{F}^{-1}(\varphi)$ so that, from Eq. (3.3),

$$
e^{2}(\varphi)=e^{2}(+\infty)\left[1-b_{F} e^{-c \varphi}\right] .
$$

The present logarithmic variation of $e^{2}$ (using again $d p$ $\left.=H d t ; \varphi^{\prime}=d \varphi / d p\right)$ is thus given by

$$
\frac{d \ln e^{2}}{H d t}=\frac{d \ln e^{2}}{d p} \simeq b_{F} c e^{-c \varphi} \varphi_{0}^{\prime},
$$

where the current value of $\varphi^{\prime}, \varphi_{0}^{\prime}$, is given in general by Eq. (2.32). Using Eq. (3.4), we can rewrite the result (3.18) in terms of the hadronic coupling:

$$
\frac{d \ln e^{2}}{H d t} \simeq \frac{1}{40} \alpha_{\text {had }} \varphi_{0}^{\prime} .
$$

As said in Sec. II B, we have basically two alternatives concerning the current coupling of the dilaton to the dominant energy sources in the universe. These two alternatives lead to drastically different predictions for the current value of the rate of variation of the fine-structure constant. We shall consider these two alternatives in turn.

In the conservative case where the dilaton does not play any special role in the present accelerated phase of the universe $\left(\alpha_{V} \simeq 0\right)$ nor does it have any stronger coupling to dark matter than to visible matter $\left(\alpha_{m} \simeq-b_{m} c e^{-c \varphi}\right)$ the dilaton "velocity" $\varphi^{\prime}$ is exponentially suppressed [so that, from Eq. (2.34), $\left.\Omega_{V} \simeq 1-\Omega_{m}\right]$ and by Eq. (2.32) one obtains

$$
\begin{aligned}
\frac{d \ln e^{2}}{H d t} & \simeq-\frac{\Omega_{m}}{\Omega_{m}+2 \Omega_{V}} b_{F} c e^{-c \varphi} \alpha_{m}(\varphi) \\
& \simeq \frac{\Omega_{m}}{2-\Omega_{m}} b_{F} b_{m} c^{2} e^{-2 c \varphi} .
\end{aligned}
$$

An indicative value for the ratio $\Omega_{m} /\left(\Omega_{m}+2 \Omega_{V}\right) \simeq \Omega_{m} /(2$ $-\Omega_{m}$ ), by taking, for instance, $\Omega_{m}=0.3$, is 0.18 . As above, it is useful to relate Eq. (3.20) to the estimate (3.4) for $\alpha_{\text {had }}$. This yields

$$
\frac{d \ln e^{2}}{H d t} \simeq \frac{1}{(40)^{2}} \frac{\Omega_{m}}{2-\Omega_{m}} \frac{b_{m}}{b_{F}} \alpha_{\text {had }}^{2} .
$$

In terms of the UFF level $\Delta a / a$ predicted by our model in Eq. (3.13) we see also that

$$
\frac{d \ln e^{2}}{H d t} \simeq 12 \frac{\Omega_{m}}{2-\Omega_{m}} \frac{b_{m}}{b_{F}} \frac{\Delta a}{a} .
$$

Even if the universe were completely dominated by dark matter $\left(\Omega_{m}=1\right)$ we see, assuming that $b_{m} / b_{F}$ is of order unity, that current experimental limits on UFF $(\Delta a / a$ $\lesssim 10^{-12}$ ) imply (within dilaton models) that $\left|d \ln e^{2} / d t\right|$ $\leq 10^{-11} H \sim 10^{-21} \mathrm{yr}^{-1}$ (the sign of $d \ln e^{2} / d t$ being given by the sign of $\left.b_{m} / b_{F}\right)$. This level of variation is much smaller than the current best limit on the time variation of $e^{2}$, namely, $\left|d \ln e^{2} / d t\right| \leq 5 \times 10^{-17} \mathrm{yr}^{-1} \sim 5 \times 10^{-7} H$, as obtained from an analysis of Oklo data [23]. (Note that the assumption-dependent analysis of Ref. [24] gives a limit on the variation of $e^{2}$ which is strengthened by about two orders of magnitude.)

The situation, however, is drastically different if we consider the alternative case where the dilaton coupling to the current dominant energy sources does not tend to triviality, as in the case of a $\varphi$-dependent vacuum energy $V(\varphi)=V_{0}$ $+V_{1} e^{-c \varphi}$ when the first term is zero or negligible. In such a case the dilaton shares a relevant part of the total energy density and more significant (though still quite constrained by UFF data) variations of the coupling constants are generally expected. A general expression for the dilaton "velocity" is given in Eq. (2.36) in terms of observable quantities. Using Eqs. (2.36) and (3.19) one can relate the expected variation of the electromagnetic coupling constant to the hadronic coupling:

$$
\frac{d \ln e^{2}}{H d t} \simeq \pm \frac{\alpha_{\text {had }}}{40} \sqrt{1+q_{0}-3 \Omega_{m} / 2} .
$$

We can also use the estimate (3.5) relating $\alpha_{\text {had }}$ to the density fluctuations generated during inflation. We obtain

$$
\frac{d \ln e^{2}}{H d t} \simeq \pm 8 \times 10^{-2} \sqrt{1+q_{0}-3 \Omega_{m} / 2} \frac{b_{F}}{b_{\lambda} c} \delta_{H}^{4 /(n+2)} .
$$

However, in view of the theoretical uncertainties attached to the initial conditions $\chi_{\text {in }}$ and $\varphi_{\text {in }}$ used in the estimate (3.5), as well as the ones associated with the order unity ratio $b_{F} /\left(b_{\lambda} c\right)$, it is more interesting to rewrite our prediction in terms of observable quantities. Using again the link Eq. (3.13) between $\alpha_{\text {had }}$ and the observable violation of the universality of free fall the above result can be written in the form

$$
\frac{d \ln e^{2}}{H d t} \simeq \pm 3.5 \times 10^{-6} \sqrt{1+q_{0}-3 \Omega_{m} / 2} \sqrt{10^{12} \frac{\Delta a}{a}} .
$$

Note that the sign of the variation of $e^{2}$ is in general model dependent (as it depends on both the sign of $b_{F}$ and the sign of $\varphi_{0}^{\prime}$ ). Specific classes of models might, however, favor particular signs of $d e^{2} / d t$. For instance, from the point of view of [5] one would expect the $\mathcal{O}\left(e^{-\phi}\right)$ terms in Eq. (1.3) to be positive, which would then imply that $b_{F}$ is positive. If we combine this information with the prediction Eq. (2.33) of the model [6] implying that $\varphi^{\prime}$ is also positive, we reach the conclusion that $e^{2}$ must be currently increasing. 
Independently of this question of the sign, we see that Eq. (3.25) predicts an interesting link between the observational violation of the UFF (constrained to $\Delta a / a \lesssim 10^{-12}$ ), and the current time variation of the fine-structure constant. Contrary to the relation (3.22), obtained above under the alternative assumption about the dilaton dependence of the dominant cosmological energy, which predicted a relation linear in $\Delta a / a$, we have here a relation involving the square root of the UFF violation (such a relation is similar to the result of [11] which concerned the time variation of the Newton constant).

The phenomenologically interesting consequence of Eq. (3.25) is to predict a time variation of constants which may be large enough to be detected by high-precision laboratory experiments. Indeed, using $H_{0} \simeq 66 \mathrm{~km} / \mathrm{s} / \mathrm{Mpc}$, and the plausible estimates $\Omega_{m}=0.3, q_{0}=-0.4$, Eq. (3.25) yields the numerical estimate $d \ln e^{2} / d t$ $\sim \pm 0.9 \times 10^{-16} \sqrt{10^{12} \Delta a / a} \mathrm{yr}^{-1}$. Therefore, the current bound on UFF violations $\left(\Delta a / a \sim 10^{-12}\right)$ corresponds to the level $10^{-16} \mathrm{yr}^{-1}$, which is comparable to the planned sensitivity of currently developed cold-atom clocks [25]. (Present laboratory bounds are at the $10^{-14} \mathrm{yr}^{-1}$ level $[26,25]$.) Note that, if we insert in Eq. (3.25) the secure bounds $\Omega_{m}>0.2$ and $q_{0}<0$ [leading to the limit Eq. (2.37)], we get as maximal estimate of the time variation of the fine-structure constant $d \ln e^{2} / d t \sim \pm 2.0 \times 10^{-16} \sqrt{10^{12} \Delta a / a} \mathrm{yr}^{-1}$. We note also that the upper limit on the variation of $e^{2}$ given by the Oklo data, i.e., $\left|d \ln e^{2} / d t\right| \leq 5 \times 10^{-17} \mathrm{yr}^{-1}$ [23], "corresponds" to a violation of the UFF at the level $\sim 10^{-13}$.

In this respect, it is interesting to consider not only the present variation of $e^{2}$ (the only one relevant for laboratory experiments), but also its variation over several billions of years. (We recall that the Oklo phenomenon took place about two billion years ago, and that astronomical observations constrain the variation of $e^{2}$ over the last ten billion years or so.) In particular, an interesting question is to see whether our model could reconcile the Oklo limit (which corresponds to a redshift $z \simeq 0.14$ ) with the recent claim [27] of a variation $\Delta e^{2} / e^{2}=(-0.72 \pm 0.18) \times 10^{-5}$ around redshifts $z$ $\approx 0.5-3.5$ as proposed in $[12,13]$. The only hope of reconciling the two results would be to allow for a faster variation of $e^{2}$ for redshifts $z>0.5$. Such recent redshifts have (apparently) been connected to a transition from matter dominance to vacuum dominance. Let us see whether taking into account this transition might allow for a large enough change of $e^{2}$ around redshifts $z \approx 0.5-3.5$. We must clearly assume the "strong coupling" scenario $\alpha_{m}=\mathcal{O}(1)$. In this scenario, the variation of $\varphi$ during the matter era is given by Eq. (2.31). Neglecting, for simplicity, the transient evolution effects localized around the matter-vacuum transition (and treating both $\varphi_{m}^{\prime}=-\alpha_{m}$ and $\varphi_{V}^{\prime}=\varphi_{0}^{\prime}$ as constants), the solution giving the recent cosmological evolution of $\varphi$ reads $\varphi$ $-\varphi_{0}=-\varphi_{0}^{\prime} \ln (1+z)$ during the vacuum era, and $\varphi-\varphi_{0}$ $=-\varphi_{0}^{\prime} \ln \left(1+z_{*}\right)-\varphi_{m}^{\prime} \ln \left[(1+z) /\left(1+z_{*}\right)\right]$ during the matter era (the index 0 refers to the present epoch, i.e., $z=0 ; z_{*}$ denotes the transition redshift). Inserting this change in Eq. (3.17) leads to the following expression for the cosmological change of the fine-structure constant:

$$
\begin{aligned}
\frac{e^{2}-e_{0}^{2}}{e_{0}^{2}}= & -\operatorname{sgn}\left(b_{F}\right) 3.5 \times 10^{-6} \\
& \times\left[\varphi_{0}^{\prime} \ln \left(1+z_{*}\right)+\varphi_{m}^{\prime} \ln \frac{1+z}{1+z_{*}}\right] \sqrt{10^{12} \frac{\Delta a}{a}} .
\end{aligned}
$$

Here, we have written the result for the matter era. During the vacuum era the bracket is simply $\left[\varphi_{0}^{\prime} \ln (1+z)\right]$. Remembering that the absolute value of $\varphi_{m}^{\prime}$ is (like that of $\varphi_{V}^{\prime}$ ) observationally constrained to be smaller than $\sqrt{0.3} \simeq 0.55$ (and that $\varphi_{0}^{\prime}$ is also constrained by $\left|\varphi_{V}^{\prime}\right|<0.84$ ), we see that there is no way, within our model, to explain a variation of $e^{2}$ as large as $\Delta e^{2} / e^{2}=(-0.72 \pm 0.18) \times 10^{-5}$ around redshifts $z \approx 0.5-3.5$ [27]. In our model, even under the assumption that UFF is violated just below the currently tested level, such a change would have to correspond to a value $\left|\varphi_{m}^{\prime}\right|$ $>2$, entailing observationally unacceptable modifications of standard cosmology. [For instance, in the model [6] a value as large as $\alpha_{m}>1$ already leads to a pathological behavior ("total dragging") where all the components scale like radiation.] This difficulty of reconciling the Oklo limit with the claim of [27] was addressed in [13,12] within a different class of models, namely, with a field $\phi$ which does not couple universally to all gauge fields $F_{\mu \nu}$, as the dilaton $\varphi$ is expected to do. The fact that the field $\phi$ in [13] (or $\psi$ in [12]) is assumed to couple only to the electromagnetic gauge field drastically changes our Eq. (3.13) and allows one to satisfy the UFF limit $\Delta a / a \leqslant 10^{-12}$ for a stronger coupling of $\phi$ to electromagnetism than in our class of models, i.e., (in our notation) for a larger $d \ln B_{F}(\varphi) / d \varphi$. This explains why Ref. [13] could construct some explicit (but fine-tuned) models in which all observational limits (UFF, Oklo, etc.) could be met and still allow for a variation of $e^{2}$ as strong as the claim [28]. The maximal variation predicted by Eq. (3.26) for redshifts corresponding to the matter era (obtained when $\Delta a / a$ $=10^{-12}$ and $\varphi_{m}^{\prime}= \pm \sqrt{0.3}$; and assuming a smaller value of $\varphi_{0}^{\prime}$ to be compatible with the Oklo constraint) is of order $\Delta e^{2} / e^{2}= \pm 1.9 \times 10^{-6}$. This is only a factor $\sim 4$ below the claim [27] and is at the level of their one sigma error bar. Therefore a modest improvement in the observational precision (accompanied by an improved control of systematics) will start to probe a domain of variation of constants which, according to our scenario, corresponds to an UFF violation smaller than the $10^{-12}$ level.

\section{Spatiotemporal fluctuations of the "constants"}

We now turn to the second possible source of spatial/ temporal variations for $e^{2}$ in our model, the quantum fluctuations of the dilaton generated during inflation. Within linear perturbation theory, the relevant calculation may be summarized as follows.

Consider a flat Friedmann-Robertson-Walker (FRW) universe $d s^{2}=-d t^{2}+a(t)^{2} \Sigma_{i} d x_{i}^{2}$. The dilaton fluctuations can be expanded in Fourier components $\delta \varphi_{\mathbf{k}}$ of given comoving momentum $\mathbf{k}$ as follows:

$$
\delta \varphi(\mathbf{x}, t)=\frac{1}{(2 \pi)^{3 / 2}} \int d^{3} k \delta \varphi_{\mathbf{k}}(t) e^{i \mathbf{k x}}
$$


where $t$ is the cosmological time. Each Fourier mode $\delta \varphi_{\mathbf{k}}$ "leaves" the horizon during inflation with an amplitude $\sim \hat{H}_{\mathrm{ex}}(k) / \sqrt{2 k^{3}}$ [29] where, by definition, $\hat{H}_{\mathrm{ex}}(k)$ is the value of the dimensionless Hubble expansion rate as $\mathrm{ka}^{-1}$ equals $H$ during inflation (note that we denote here by $\hat{H}_{\mathrm{ex}}$ what was denoted by $\hat{H}_{\times}$above). Well after the exit $(k \ll a H)$ the am- plitude of each mode "freezes out," i.e., remains roughly constant, until it reenters the horizon during the postinflationary epoch $\left(k a_{r e}^{-1} \simeq H_{r e}\right)$. After reentry the amplitude starts to damp out as $a^{-1}$. For a given Fourier mode $\delta \varphi_{\mathbf{k}}(t)$, the latter damping effect is described by the piecewise function

$$
f_{z}(k) \equiv\left\{\begin{array}{lll}
1 & \text { if } & a_{0}^{-1} H_{0}^{-1} k<(z+1)^{1 / 2}, \\
a_{0}^{2} H_{0}^{2}(z+1) k^{-2} & \text { if } \quad(z+1)^{1 / 2}<a_{0}^{-1} H_{0}^{-1} k<10^{2}, \\
10^{-2} a_{0} H_{0}(z+1) k^{-1} & \text { if } \quad a_{0}^{-1} H_{0}^{-1} k>10^{2} .
\end{array}\right.
$$

Here the cosmological redshift $z=a_{0} / a(t)-1$ has been introduced in replacement of the cosmological time $t$. The first case refers to Fourier modes that have not reentered yet at redshift $z$ and whose amplitudes are still frozen. The second and third cases refer to modes that reenter during matter and radiation domination, respectively. Putting all together, and assuming a Gaussian probability distribution for the perturbations, we have

$$
\left\langle\delta \varphi_{\mathbf{k}}(t)^{*} \delta \varphi_{\mathbf{k}^{\prime}}\left(t^{\prime}\right)\right\rangle=\frac{\hat{H}_{\mathrm{ex}}^{2}(k)}{2 k^{3}} f_{z}(k) f_{z^{\prime}}(k) \delta^{3}\left(\mathbf{k}-\mathbf{k}^{\prime}\right) .
$$

Possible spatial/temporal variations of $e^{2}$ induced by the fluctuations of the dilaton will be given by

$$
\left.\frac{\Delta^{\text {fluc }} e^{2}}{e^{2}}\right|_{\left(\mathbf{x}, t ; \mathbf{x}^{\prime}, t^{\prime}\right)}=\left.\frac{d \ln e^{2}}{d \varphi} \Delta^{\text {fluc }} \varphi\right|_{\left(\mathbf{x}, t ; \mathbf{x}^{\prime}, t^{\prime}\right)},
$$

where the rms $\Delta^{\text {fluc }} \varphi$ between two events $(\mathbf{x}, t)$ and $\left(\mathbf{x}^{\prime}, t^{\prime}\right)$ is defined as follows:

$$
\begin{aligned}
\left.\Delta^{\text {fluc }} \varphi\right|_{\left(\mathbf{x}, t ; \mathbf{x}^{\prime}, t^{\prime}\right)} ^{2} & \equiv\left\langle\left[\delta \varphi(\mathbf{x}, t)-\delta \varphi\left(\mathbf{x}^{\prime}, t^{\prime}\right)\right]^{2}\right\rangle \\
& =\frac{\hat{H}_{\mathrm{ex}}^{2}}{(2 \pi)^{3}} \int \frac{d^{3} k}{2 k^{3}}\left[f_{z}(k)^{2}+f_{z^{\prime}}(k)^{2}-2 f_{z}(k) f_{z^{\prime}}(k) e^{i \mathbf{k}\left(\mathbf{x}-\mathbf{x}^{\prime}\right)}\right] \\
& =\frac{\hat{H}_{\mathrm{ex}}^{2}}{(2 \pi)^{2}} \int_{0}^{\infty} \frac{d k}{k}\left\{\left[f_{z}(k)-f_{z^{\prime}}(k)\right]^{2}+2 f_{z}(k) f_{z^{\prime}}(k)\left[1-\frac{\sin k x}{k x}\right]\right\} .
\end{aligned}
$$

Here, $x \equiv\left|\mathbf{x}-\mathbf{x}^{\prime}\right|$ is the coordinate distance between the two events and, consistently with the slow-roll approximation, the Hubble expansion rate at exit has been assumed to be scale invariant: $\hat{H}_{\mathrm{ex}}(k) \simeq \hat{H}_{\mathrm{ex}} \simeq 3 \times 10^{-5}$.

If one considers spatial fluctuations over terrestrial or solar-system proper length scales $l=a_{0} k^{-1} \ll H_{0}^{-1}$ at the present time $t=t^{\prime}=t_{0}$, the first square brackets in Eq. (3.32) vanishes and one can expand the sine function at small $k x$, obtaining
As expected, these variations are extremely small, $\Delta^{\text {fluc }} e^{2} /\left.e^{2}\right|_{l ; z=0} \simeq 10^{-33} l / \mathrm{km}$. It is also interesting to compare dilaton fluctuations at different redshifts along a comoving observer worldline. By putting $x \simeq 0$ in Eq. (3.32) the second term in the square brackets vanishes and one has

$$
\begin{aligned}
\left.\Delta^{\mathrm{fluc}} \varphi\right|_{z ; x=0} & \simeq \frac{\hat{H}_{\mathrm{ex}}}{2 \pi} \frac{1}{\sqrt{2}}\left[\log (1+z)-\frac{z}{1+z}+\frac{10^{-8}}{2} z^{2}\right]^{1 / 2} \\
& \simeq \frac{\hat{H}_{\mathrm{ex}}}{2 \pi}\left[\frac{z}{2}-\frac{z^{2}}{3}+\cdots\right]
\end{aligned}
$$

It is slightly more complicated to compare dilaton fluctuations between "now" and events at redshift $z$ along a null 
ray. Expanding in powers of $z$ around $z=0$ one gets from Eq. (3.32), after a straightforward calculation,

$$
\left.\Delta^{\text {fluc }} \varphi\right|_{z} \simeq \frac{\hat{H}_{\mathrm{ex}}}{2 \pi}\left[\frac{1}{2} \sqrt{\frac{7}{3}} z-\frac{2}{\sqrt{21}} z^{2}+\cdots\right] .
$$

Numerically, at redshift $z \sim 1$, the effects of dilatonic fluctuations give $\left.\Delta^{\text {fluc }} \varphi\right|_{z=1} \sim \hat{H}_{\text {ex }} /(2 \pi) \sim 5 \times 10^{-6}$. This is to be contrasted with the effects of the cosmic, homogeneous evolution which yields $\left.\Delta \varphi\right|_{z=1} \simeq \alpha_{m}$. In the "normal" case where $\alpha_{m} \sim e^{-c \varphi} \sim \delta_{H}^{4 /(n+2)}$, the two effects, though a priori unrelated, are related in our scenario, when $n=2$. Indeed, if $n=2, \quad \delta_{H}^{4 /(n+2)}=\delta_{H} \sim 5 \times 10^{-5}$ is linked to $\hat{H}_{\mathrm{ex}} /(2 \pi)$ via $\delta\left(\chi_{\mathrm{ex}}\right)=A \hat{H}_{\mathrm{ex}} /(2 \pi) \quad$ with $\quad A=(8 / 3) V / \partial_{\chi} V=(8 / 3)(\chi / n)$ $\simeq 40 /(3 \sqrt{n}) \sim 10$. On the other hand, in the case where $\varphi$ is strongly coupled to dark matter, the homogeneous evolution $\left.\Delta \varphi\right|_{z=1} \simeq \alpha_{m} \sim 1$ is parametrically larger than the fluctuations $\left.\Delta^{\text {fluc }} \varphi\right|_{z=1} \sim \hat{H}_{\mathrm{ex}} /(2 \pi)$.

To conclude this subsection, we see that the inhomogeneous space-time fluctuations of the fine-structure constant are typically too small to be observable (if the limits from UFF are already satisfied), being suppressed, relative to their natural values $H_{0} l, H_{0} t$, by the small factor $\alpha_{\text {had }} \hat{H}_{\text {ex }}$.

\section{SUMMARY AND CONCLUSION}

We have studied the dilaton-fixing mechanism of [3] within the context where the dilaton-dependent low-energy couplings are extremized at $\varphi=+\infty$, i.e., for infinitely large values of the bare string coupling $g_{s}^{2}=e^{\phi} \simeq e^{c \varphi}$. [The crucial coupling to the inflaton, say $\lambda(\varphi)$ in Eq. (2.11), must be minimized at $\varphi \rightarrow+\infty$; the other couplings can be either minimized or maximized there.] This possibility of a fixed point at infinity (in bare string coupling space) has recently been suggested [5], and its late cosmological consequences have been explored in [6]. We found that a primordial inflationary stage, with inflaton potential $V(\chi)=\lambda(\varphi) \chi^{n} / n$, was much less efficient in decoupling a dilaton with least couplings at infinity than in the case where the least couplings are reached at a finite value of $\varphi$ (as in [3,8]). This reduced efficiency has interesting phenomenological consequences. Indeed, it predicts much larger observable deviations from general relativity. In the case of the simplest chaotic potential [10] $V(\chi)=\frac{1}{2} m_{\chi}^{2}(\varphi) \chi^{2}$, we find that, under the simplest assumptions about the preinflationary state, this scenario predicts violations of the universality of free fall of order $\Delta a / a \sim 5 \times 10^{-4} \delta_{H}^{2}$ where $\delta_{H}$ is the density fluctuation generated by inflation on horizon scales. The observed level of large-scale density (and cosmic microwave background temperature) fluctuations fixes $\delta_{H}$ to be around $5 \times 10^{-5}$ which finally leads to a prediction for a violation of the UFF near the $\Delta a / a \sim 10^{-12}$ level. This is naturally compatible with present experimental tests of the equivalence principle, and suggests that a modest improvement in the precision of UFF tests might be able to detect a deviation linked to dilaton exchange with a coupling reduced by the attraction toward the fixed point at infinity. Because of the presence of un- known dimensionless ratios $\left(c, b_{F} / b_{\lambda}\right)$ in our estimates, and of quantum noise in the evolution of the dilaton, we cannot give sharp quantitative estimates of $\Delta a / a$. However, we note that dilaton-induced violations of the UFF have a rather precise signature with a composition dependence of the form (3.11), with probable domination by the last (Coulomb energy) term [3]. As explored in [21] this signature is quite distinct from UFF violations induced by other fields, such as a vector field. We note that the approved Center National d'Etudes Spatiales (CNES) mission MICROSCOPE [30] (to fly in 2004) will explore the level $\Delta a / a \sim 10^{-15}$, while the planned National Aeronautics and Space Agency (NASA) and European Space Agency (ESA) mission STEP (satellite test of the equivalence principle) [31] could explore the $\Delta a / a \sim 10^{-18}$ level. Our scenario gives additional motivation for such experiments and suggests that they might find a rather strong violation signal, whose composition dependence might then be studied in detail to compare it with Eq. (3.11).

In the case of inflationary potentials $V(\chi) \propto \chi^{n}$ with $n$ $>2$ our simplest estimates predict a violation of the UFF of order $\Delta a / a \sim 5 \times 10^{-4} \delta_{H}^{8 /(n+2)}$ which is larger than $10^{-12}$. At face value this suggests that existing UFF experimental data can be interpreted as favoring $n \leqslant 2$ over $n>2$. However, we must remember that our estimates have made several simplifying assumptions. It is possible that the large quantum fluctuations of the inflaton in the self-regenerating regime $\chi$ $>\chi_{\text {in }}$, with $\chi_{\text {in }}$ defined by Eq. (2.18), can give more time for $\varphi$ to run away toward large values, so that the effective value of $e^{c \varphi_{\text {in }}}$ to be used in Eq. (2.21) turn out to dominate the first term in the RHS that we have used for our estimates. We leave to future work a study of the system of Langevin equations describing the coupled fluctuations of $\phi$ and $\chi$ during the self-regenerating regime.

Finally let us note some other conclusions of our work.

We recover the conclusion of previous works on dilaton models that the most interesting experimental probes of a massless weakly coupled dilaton are tests of the UFF. The composition-independent gravitational tests (solar system, binary pulsar) tend to be much less sensitive probes [as highlighted by the relations (3.14), (3.21), and (3.22)].

However, a possible exception concerns the time variation of the coupling constants. Here the conclusion depends crucially on the assumptions made about the couplings of the dilaton to the cosmologically dominant forms of energy (dark matter and/or dark energy). If these couplings are of order unity [and as large as is phenomenologically acceptable, i.e., so that $\left.\left(\varphi_{0}^{\prime}\right)^{2}=0.7\right]$, the present time variation of the fine-structure constant is linked to the violation of the UFF by the relation $d \ln e^{2} / d t$ $\sim \pm 2.0 \times 10^{-16} \sqrt{10^{12} \Delta a / a} \mathrm{yr}^{-1}$. (The most natural sign here is + , i.e., $b_{F}>0$, which corresponds to smaller $e^{2}$ in the past, just as suggested by the claim [27].) Such a time variation might be observable (if $\Delta a / a$ is not very much below its present upper bound $\sim 10^{-12}$ ) through the comparison of high-accuracy cold-atom clocks and/or via improved measurements of astronomical spectra.

More theoretical work is needed to justify the basic as- 
sumption (1.3) of our scenario. In particular, it is crucial to investigate whether it is natural to expect that the sign of the crucial coefficient $b_{\lambda}$ in Eq. (2.12) be indeed positive. [Recall that the general mechanism of [3] is an attraction toward "least couplings" while Eq. (1.3) with $\mathcal{O}\left(e^{-\phi}\right)>0$ leads to largest couplings at infinity.] Note in this respect that the sign of the other $b_{i}$ 's is not important as, once inflation has pushed $e^{c \varphi}$ to very large values $e^{c \varphi}$ end, the subsequent cosmological evolutions tend to be ineffective in further displacing $\varphi$.

\section{ACKNOWLEDGMENTS}

It is a pleasure to thank Ian Kogan for suggesting a method to solve the (decoupled) Langevin equation for $\varphi$. G.V. wishes to acknowledge the support of a "Chaire Internationale Blaise Pascal," administered by the "Fondation de L'Ecole Normale Supérieure." The work of F.P. was supported in part by INFN and MURST under contract 2001025492, and by the European Commission TMR program HPRN-CT-2000-00131, in association to the University of Padova.

\section{APPENDIX: THE STOCHASTIC EVOLUTION OF THE DILATON}

In this appendix we study the stochastic evolution of the dilaton $\varphi$ during inflation as described by the Langevin-type equation (2.23). We restrict our attention to the region of phase space where the evolution of the inflaton $\chi$ is classical, and to a power-law potential of the form (2.11). It follows that the inflaton evolves according to the classical slow-roll equation (2.14) whose solution reads

$$
\chi^{2}=\chi_{\text {in }}^{2}-n p,
$$

where $p$, the parameter defined in Eq. (2.7), is shifted in such a way that $p_{\text {in }} \equiv 0$. Equation (2.23) takes the form

$$
\frac{d \varphi}{d p}=\frac{1}{2} b_{\lambda} c e^{-c \varphi}+\xi(p),
$$

where $\xi(p)$ is a Gaussian stochastic variable (GSV), with a "time-dependent" rms amplitude $\hat{H}(p) / 2 \pi$ :

$$
\left\langle\xi\left(p_{1}\right) \xi\left(p_{2}\right)\right\rangle=\frac{\hat{H}^{2}}{(2 \pi)^{2}} \delta\left(p_{1}-p_{2}\right)
$$

[the relation to the normalized random white noise term of Eq. (2.23) is $\left.\xi(p)=\xi_{2}(p) \hat{H} / 2 \pi\right]$. For any given source term $\xi(p)$, the formal solution of Eq. (A2) reads

$$
\begin{aligned}
e^{c \varphi(p)} & =e^{c \varphi_{\text {in }}} e^{c \eta(p)}+\frac{b_{\lambda} c^{2}}{2} \int_{0}^{p} d p^{\prime} e^{c\left[\eta(p)-\eta\left(p^{\prime}\right)\right]}, \\
\eta(p) & \equiv \int_{0}^{p} d p^{\prime} \xi\left(p^{\prime}\right) .
\end{aligned}
$$

Note that the classical solution in Eq. (2.17), $e^{c \varphi_{\mathrm{cl}}(p)}=e^{c \varphi_{\text {in }}}$ $+\left(b_{\lambda} c^{2} / 2\right) p$, can be easily recovered in the small noise limit $\xi(p) \rightarrow 0, \quad \eta(p) \rightarrow 0$.

It proves convenient to compare the true solution to the classical one by studying the statistical behavior of the ratio $A(p) \equiv e^{c \varphi(p)} / e^{c \varphi_{\mathrm{cl}}(p)}$. As we will show below, $\left\langle e^{c \eta(p)}\right\rangle$ $=\mathcal{O}(1)$. Moreover, we are also assuming $e^{c \varphi_{\text {in }}}=\mathcal{O}(1)$ or, at

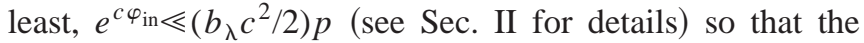
leading contribution to the first equation in (A4) is given by the integral, and we have

$$
A(p) \equiv e^{c \varphi(p)} / e^{c \varphi_{\mathrm{cl}}(p)} \simeq \frac{1}{p} \int_{0}^{p} d p^{\prime} e^{c\left[\eta(p)-\eta\left(p^{\prime}\right)\right]} .
$$

Since $\xi(p)$ is a GSV, its integral $\eta(p)$ is also a (centered) GSV. Moreover, if $x$ is a GSV with $\sigma_{x}^{2} \equiv\left\langle x^{2}\right\rangle-\langle x\rangle^{2}=\left\langle x^{2}\right\rangle$, by Bloch's theorem $y=e^{x}$ is a new stochastic variable with $\langle y\rangle=\left\langle e^{x}\right\rangle=e^{\left\langle x^{2}\right\rangle / 2}$ and $\sigma_{y}^{2}=e^{2\left\langle x^{2}\right\rangle}-e^{\left\langle x^{2}\right\rangle}$. The average value of $A(p)$ thus reads

$$
\langle A(p)\rangle \simeq \frac{1}{p} \int_{0}^{p} d p^{\prime} e^{\left(c^{2} / 2\right)\left\langle\left[\eta(p)-\eta\left(p^{\prime}\right)\right]^{2}\right\rangle} .
$$

The exponent on the right-hand side of the above equation can be estimated by using Eq. (A3) and the slow-roll approximation $\quad \hat{H}^{2} \simeq 2 V(\chi, \varphi) / 3=2 \lambda(\varphi) \chi^{n} / 3 n \simeq 2 \lambda_{\infty} \chi^{n} / 3 n$. One gets

$$
\begin{aligned}
\left\langle\left[\eta(p)-\eta\left(p^{\prime}\right)\right]^{2}\right\rangle & =\frac{1}{(2 \pi)^{2}} \int_{p^{\prime}}^{p} \hat{H}^{2} d p^{\prime \prime} \\
& \simeq \frac{n}{2(n+2)}\left[\left(\frac{\chi\left(p^{\prime}\right)}{\chi_{\text {in }}}\right)^{n+2}-\left(\frac{\chi(p)}{\chi_{\text {in }}}\right)^{n+2}\right],
\end{aligned}
$$

where $\chi_{\text {in }}$ is the value at exit from self-regenerating inflation: $\hat{H}\left(\chi_{\text {in }}\right) / 2 \pi=n /\left(2 \chi_{\text {in }}\right)$ (see Sec. II for more details). Since we are interested in evaluating Eq. (A6) at the end of inflation, $p=p_{\text {end }} \simeq \chi_{\text {in }}^{2} / n$, we can thus write

$$
\left\langle\left[\eta(p)-\eta\left(p^{\prime}\right)\right]^{2}\right\rangle \simeq \frac{n}{2(n+2)}\left[1-\frac{p^{\prime}}{p_{\text {end }}}\right]^{(n+2) / 2} .
$$

When evaluated at $p^{\prime}=0$, the above formula gives $\left\langle\eta(p)^{2}\right\rangle=n /[2(n+2)]$. Thus the normalization factor to the initial condition in Eq. (A4) is of order 1, as anticipated: $\left\langle e^{c \eta(p)}\right\rangle=e^{(1 / 2) c\left\langle\eta(p)^{2}\right\rangle}=\mathcal{O}(1)$. From Eqs. (A6) and (A8) we have

$$
\begin{aligned}
\left\langle A\left(p_{\text {end }}\right)\right\rangle & \simeq \frac{1}{p_{\text {end }}} \int_{0}^{p_{\text {end }}} d p^{\prime} \exp \left[\frac{c^{2} n}{4(n+2)}\left(1-\frac{p^{\prime}}{p_{\text {end }}}\right)^{(n+2) / 2}\right] \\
& =\int_{0}^{1} \exp \left[\frac{c^{2} n}{4(n+2)} x^{(n+2) / 2}\right] d x \\
& =\exp \left(\frac{c^{2} n \theta}{4(n+2)}\right)=\mathcal{O}(1)
\end{aligned}
$$


with $0<\theta<1$.

We can estimate the dispersion of the same quantity by expanding the exponential inside the integral (A6) in powers of $\xi(p)$ :

$$
\begin{aligned}
A(p) \simeq 1 & +\frac{c}{p} \int_{0}^{p} d p^{\prime} \int_{p^{\prime}}^{p} d p^{\prime \prime} \xi\left(p^{\prime \prime}\right) \\
& +\frac{1}{2} \frac{c^{2}}{p} \int_{0}^{p} d p^{\prime}\left(\int_{p^{\prime}}^{p} d p^{\prime \prime} \xi\left(p^{\prime \prime}\right)\right)^{2}+\cdots
\end{aligned}
$$

At lowest order in $\xi(p)$ the variance of the above quantity calculated at $p=p_{\text {end }}$ reads

$$
\sigma_{A\left(p_{\text {end }}\right)}^{2}=\left\langle\left(\frac{c}{p} \int_{0}^{p} d p^{\prime} \int_{p^{\prime}}^{p} d p^{\prime \prime} \xi\left(p^{\prime \prime}\right)\right)^{2}\right\rangle
$$

$$
\begin{aligned}
& =\frac{c^{2}}{p^{2}} \int_{0}^{p} d p^{\prime} \int_{0}^{p} d p^{\prime \prime} \int_{\max \left(p^{\prime}, p^{\prime \prime}\right)}^{p} d p^{\prime \prime \prime} \frac{\hat{H}^{2}\left(p^{\prime \prime \prime}\right)}{(2 \pi)^{2}} \\
& =\frac{2 c^{2}}{p^{2}} \int_{0}^{p} d p^{\prime} p^{\prime} \int_{p^{\prime}}^{p} d p^{\prime \prime} \frac{\hat{H}^{2}\left(p^{\prime \prime}\right)}{(2 \pi)^{2}} .
\end{aligned}
$$

As in Eq. (A7) we can use the slow-roll approximation and obtain

$$
\begin{aligned}
\sigma_{A\left(p_{\text {end }}\right)}^{2} & =\frac{c^{2} n}{n+2} \int_{0}^{1} x(1-x)^{(n+2) / 2} d x \\
& =c^{2} \mathcal{O}(1) .
\end{aligned}
$$

[1] E. Witten, Phys. Lett. 149B, 351 (1984).

[2] T.R. Taylor and G. Veneziano, Phys. Lett. B 213, 450 (1988).

[3] T. Damour and A.M. Polyakov, Nucl. Phys. B423, 532 (1994); Gen. Relativ. Gravit. 26, 1171 (1994).

[4] T. Damour and K. Nordtvedt, Phys. Rev. Lett. 70, 2217 (1993); Phys. Rev. D 48, 3436 (1993).

[5] G. Veneziano, J. High Energy Phys. 06, 051 (2002).

[6] M. Gasperini, F. Piazza, and G. Veneziano, Phys. Rev. D 65, 023508 (2002).

[7] T. Damour, F. Piazza, and G. Veneziano, Phys. Rev. Lett. 89, 081601 (2002).

[8] T. Damour and A. Vilenkin, Phys. Rev. D 53, 2981 (1996).

[9] T. Damour, in Susy 95, Proceedings of the 1995 International Workshop on Supersymmetry and Unification of Fundamental Interactions, edited by I. Antoniadis and H. Videau (Editions Frontières, Gif-sur-Yvette, 1996), pp. 577-584.

[10] A. Linde, Particle Physics and Inflationary Cosmology (Harwood, Chur, Switzerland, 1990).

[11] T. Damour, G.W. Gibbons, and C. Gundlach, Phys. Rev. Lett. 64, 123 (1990).

[12] H.B. Sandvik, J.D. Barrow and J. Magueijo, Phys. Rev. Lett. 88, 031302 (2002).

[13] K.A. Olive and M. Pospelov, Phys. Rev. D 65, 085044 (2002).

[14] L. Amendola and D. Tocchini-Valentini, Phys. Rev. D 64, 043509 (2001).

[15] L. Amendola, M. Gasperini, C. Ungarelli, and D. TocchiniValentini (in preparation).

[16] S. Perlmutter et al., Astrophys. J. 517, 565 (1999); A. Riess et al., Astron. J. 116, 1009 (1998).

[17] See, e.g., the review of global cosmological parameters (Chap. 17) in D.E. Groom et al., Eur. Phys. J. C 15, 1 (2000); updated version available on http://pdg.lbl.gov/.
[18] D.E. Groom et al., [17], Chap. 14, available on http://pdg.lbl.gov/; C.M. Will, Living Rev. Relativ. 4, 4 (2001).

[19] T. Damour and G. Esposito-Farèse, Class. Quantum Grav. 9, 2093 (1992).

[20] T. Damour and G. Esposito-Farèse, Phys. Rev. D 54, 1474 (1996); 58, 042001 (1998).

[21] T. Damour, Class. Quantum Grav. 13, A33 (1996).

[22] Y. Su et al., Phys. Rev. D 50, 3614 (1994).

[23] A.I. Shlyakhter, Nature (London) 264, 340 (1976); T. Damour and F. Dyson, Nucl. Phys. B480, 37 (1996).

[24] K.A. Olive, M. Pospelov, Y.Z. Qian, A. Coc, M. Casse, and E. Vangioni-Flam, "Constraints on the Variations of the Fundamental Couplings," hep-ph/0205269.

[25] C. Salomon et al., in Cold Atom Clocks on Earth and in Space, Proceedings of the 17th International Conference on Atomic Physics, edited by E. Arimondo and M. Inguscio (World Scientific, Singapore, 2001), p. 23; Y. Sortais et al., Phys. Scr. 95, 50 (2001).

[26] J.D. Prestage, R.L. Tjoelker, and L. Maleki, Phys. Rev. Lett. 74, 3511 (1995).

[27] J.K. Webb et al., Phys. Rev. Lett. 87, 091301 (2001).

[28] M. Gasperini, Phys. Rev. D 64, 043510 (2001).

[29] A.R. Liddle and D.H. Lyth, Cosmological Inflation and Large Scale Structure (Cambridge University Press, Cambridge, England, 2000).

[30] P. Touboul et al., C. R. Acad. Sci. Ser. IV: Astrophys. 2, 1271 (2001).

[31] P.W. Worden, in Proceedings of the Seventh Marcel Grossmann Meeting on General Relativity, edited by R.T. Jantzen and G. Mac Keiser (World Scientific, Singapore, 1996), pp. $1569-1573$. 